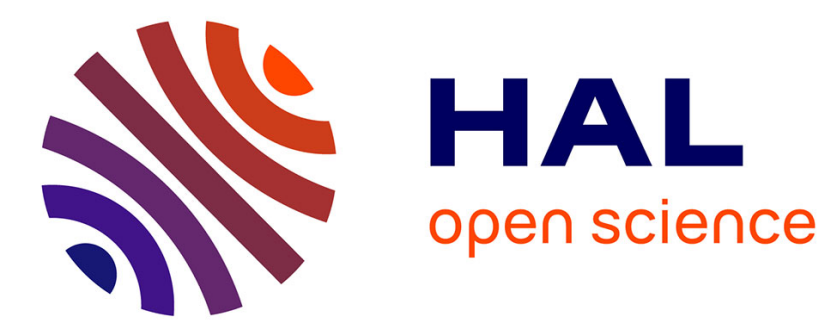

\title{
Bose condensates in interaction with excitations - a kinetic model
}

Leif Arkeryd, Anne Nouri

\section{To cite this version:}

Leif Arkeryd, Anne Nouri. Bose condensates in interaction with excitations - a kinetic model. Communications in Mathematical Physics, 2012, 310 (3), pp.765-788. hal-00824011

\section{HAL Id: hal-00824011 \\ https://hal.science/hal-00824011}

Submitted on 20 May 2013

HAL is a multi-disciplinary open access archive for the deposit and dissemination of scientific research documents, whether they are published or not. The documents may come from teaching and research institutions in France or abroad, or from public or private research centers.
L'archive ouverte pluridisciplinaire HAL, est destinée au dépôt et à la diffusion de documents scientifiques de niveau recherche, publiés ou non, émanant des établissements d'enseignement et de recherche français ou étrangers, des laboratoires publics ou privés. 


\title{
Bose condensates in interaction with excitations - a kinetic model.
}

\author{
Leif ARKERYD and Anne NOURI \\ Chalmers, 41296 Göteborg, Sweden, \\ LATP, Aix-Marseille University, France
}

\begin{abstract}
This paper deals with mathematical questions for Bose gases below the temperature $T_{B E C}$ where Bose-Einstein condensation sets in. The model considered is of two-component type, consisting of a kinetic equation for the distribution function of a gas of (quasi-)particles interacting with a Bose condensate, which is described by a Gross-Pitaevskii equation. Existence results and moment estimates are proved in the space-homogeneous, isotropic case.
\end{abstract}

\section{Preliminaries.}

Starting from a detailed effective Hamiltonian for a Bose fluid, and motivated by considerations about the relevant physics, simplified models for classical fields can be derived in well-defined (formal) limits (cf [BCEP, S1]), or obtained by physics arguments for more direct approximations of the original potentials and operators. There for sufficiently low temperatures only interactions between thermally excited (quasi-)particles and condensate are of practical importance, as discussed in the papers [K, KK, HM, E, KD, ZNG, IT, ITG] and their references.

The present paper considers one such situation involving transfer of atoms between the two components, so that in particular no conservation laws for an individual component should be expected in the transient evolution. The model is based on the Beliaev-Popov approximation of the Bogoliubov Green-function description which ignores off-diagonal correlations, and the Thomas-Fermi approximation which neglects a quantum pressure term. The simplified two-component model obtained, consists of a kinetic equation for the distribution function of a gas of (quasi-)particles interacting with a Bose condensate, which in turn is described by a Gross-Pitaevskii equation (cf [PS]). In the local rest frame, the kinetic equation is ([ITG])

$$
\frac{\partial f}{\partial t}+\nabla_{p}\left(E_{p}+v_{s} p\right) \cdot \nabla_{x} f-\nabla_{x}\left(E_{p}+v_{s} p\right) \cdot \nabla_{p} f=C\left(f, n_{c}\right) .
$$

Here $v_{s}$ is the superfluid velocity, $E_{p}$ the (Bogoliubov) excitation energy, and the collision term becomes

$$
\begin{array}{r}
C\left(f, n_{c}\right)(p)=n_{c} \int|A|^{2} \delta\left(p_{1}-p_{2}-p_{3}\right) \delta\left(E_{1}-E_{2}-E_{3}\right)\left[\delta\left(p-p_{1}\right)\right. \\
\left.-\delta\left(p-p_{2}\right)-\delta\left(p-p_{3}\right)\right]\left(\left(1+f_{1}\right) f_{2} f_{3}-f_{1}\left(1+f_{2}\right)\left(1+f_{3}\right)\right) d p_{1} d p_{2} d p_{3} .
\end{array}
$$

The transition probability kernel $|A|^{2}$ is given by the scattering amplitude

$$
A:=\left(u_{3}-v_{3}\right)\left(u_{1} u_{2}+v_{1} v_{2}\right)+\left(u_{2}-v_{2}\right)\left(u_{1} u_{3}+v_{1} v_{3}\right)-\left(u_{1}-v_{1}\right)\left(u_{2} v_{3}+v_{2} u_{3}\right) .
$$

\footnotetext{
${ }^{1} 2010$ Mathematics Subject Classification. 82C10, 82C22, 82C40.

${ }^{2}$ Key words; low temperature kinetics, Bose condensate, two component model, below $0.7 T_{B E C}$.
} 
Here the Bose coherence factors $u$ and $v$ are

$$
u_{p}^{2}=\frac{\tilde{\epsilon}_{p}+E_{p}}{2 E_{p}}, \quad u_{p}^{2}-v_{p}^{2}=1
$$

with $\tilde{\epsilon}_{p}=\frac{p^{2}}{2 m}+g n_{c}, n_{c}$ the non-equilibrium density of the atoms in the condensate, $m$ the atomic mass, and $g$ a scattering length defined later.

The collision operator $C\left(f, n_{c}\right)$ can be formally obtained (cf [ST], [EMV], [N]) from the NordheimUehling-Uhlenbeck collision operator

$$
\begin{array}{r}
\tilde{C}_{N U U}(f)(p)=\int_{\mathbb{R}^{3} \times \mathbb{R}^{3} \times \mathbb{R}^{3}} B \delta\left(p+p_{*}=p^{\prime}+p_{*}^{\prime}\right) \delta\left(E(p)+E\left(p_{*}\right)=E\left(p^{\prime}\right)+E\left(p_{*}^{\prime}\right)\right) \\
\left(f^{\prime} f_{*}^{\prime}(1+f)\left(1+f_{*}\right)-f f_{*}\left(1+f^{\prime}\right)\left(1+f_{*}^{\prime}\right)\right) d p_{*} d p^{\prime} d p_{*}^{\prime}
\end{array}
$$

with

$$
f_{*}=f\left(p_{*}\right), \quad f^{\prime}=f\left(p^{\prime}\right), \quad f_{*}^{\prime}=f\left(p_{*}^{\prime}\right) .
$$

Assuming that a condensate appears below the Bose-Einstein condensation temperature $T_{B E C}$, which splits the quantum gas distribution function into a condensate part $n_{c} \delta_{p=0}$ and an $L^{1}$-density part $f(t, x, p)$, we obtain

$$
\tilde{C}_{N U U}\left(f+n_{c} \delta_{p=0}\right)=\tilde{C}_{N U U}(f)+C\left(f, n_{c}\right)+n_{c}^{2} \tilde{A}+n_{c}^{3} \tilde{B}+n_{c} \delta_{p=0} \tilde{D},
$$

where a simple formal computation shows that $\tilde{A}=\tilde{B}=0$. At the low temperatures considered, the number of excited (quasi-)particles is small, and the $\tilde{C}_{N U U}$ collision term can be neglected relative to the collision operator $C$.

The usual Gross-Pitaevskii (GP) equation for the wave function $\psi$ (the order parameter) associated with a Bose condensate is

$$
i h \frac{\partial \psi}{\partial t}=-\frac{h^{2}}{2 m} \Delta_{x} \psi+\left(U_{e x t}+g|\psi|^{2}\right) \psi
$$

i.e. a Schrödinger equation complemented by a non-linear term accounting for two-body interactions. $U_{\text {ext }}$ is an external potential, and with $a$ the $s$-scattering length of the interaction potential and $g=\frac{4 \pi a}{m}$. In the present context the GP equation is further generalized by letting the condensate move in a self-consistent Hartree-Fock mean field $2 g \tilde{n}=2 g \int f(p) d p$ produced by the thermally excited atoms, together with a dissipative coupling term associated with the collisions. It is useful to split the equation for $\psi=\sqrt{n_{c}} e^{i \theta}$ into phase and amplitude variables (polar representation or the Madelung transform, cf [ZNG]), leading to

$$
\begin{aligned}
& \frac{\partial n_{c}}{\partial t}+\nabla x \cdot\left(n_{c} v_{s}\right)=-\int C\left(f, n_{c}\right) d p \\
& \frac{\partial \theta}{\partial t}=-\mu_{c}-\frac{m v_{s}^{2}}{2}
\end{aligned}
$$

with $\mu_{c}$ a local condensate chemical potential. 


\section{A space-homogeneous isotropic case; the mathematical setting.}

This paper is the first part of a study of the Cauchy problem for the two component model (1.1), (1.3) of a kinetic gas of quasi-particles interacting with a GP condensate. The focus is on the spacehomogeneous isotropic case and the superfluid rest frame (condensate velocity $v_{s}=\nabla_{x} \theta=0$ ), i.e. the equations

$$
\begin{array}{r}
\frac{\partial f}{\partial t}=C\left(f, n_{c}\right), \\
\frac{d n_{c}}{d t}=-\int C\left(f, n_{c}\right) d p,
\end{array}
$$

with initial values

$$
f(p, 0)=f_{i}(|p|), \quad n_{c}(0)=n_{c i} .
$$

Here $f(p, t)$ is the density of the quasi-particles, $n_{c}(t)$ the mass of the condensate, and the collision operator $C$ is given by (1.2).

The papers [E, ITG] consider the low temperature situation where the temperature is smaller than $0.4 T_{B E C}$, with all $\left|p_{i}\right|<<p_{0}$, i.e. where physically all quasi-particle momenta are much smaller than the characteristic momentum $p_{0}=\sqrt{2 m g n_{c}}$ for the crossover between the linear and quadratic parts of the Bogoliubov excitation energy of the quasi-particles;

$$
E(p):=\sqrt{\frac{p^{4}}{4 m^{2}}+\frac{g n_{c}}{m} p^{2}} \approx c|p|\left(1+\frac{p^{2}}{8 g m n_{c}}\right)=c|p|\left(1+\frac{p^{2}}{4 p_{0}^{2}}\right)
$$

with $c:=\sqrt{\frac{g n_{c}}{m}}$ the speed of Bogoliubov sound. Setting $m=\frac{1}{\sqrt{2}}$ gives $p_{0}=c$.

The right hand side of (2.4) is usually taken as the value of $E(p)$ in applications with $|p|<<p_{0}$. The Bose coherence factors can then be taken as

$$
u_{p}=\sqrt{\frac{g n_{c}}{2 E(p)}}+\frac{1}{2} \sqrt{\frac{E(p)}{2 g n_{c}}}, \quad v_{p}=\sqrt{\frac{g n_{c}}{2 E(p)}}-\frac{1}{2} \sqrt{\frac{E(p)}{2 g n_{c}}}, \quad u_{p}^{2}-v_{p}^{2}=1,
$$

which gives

$$
A=\frac{1}{2^{\frac{5}{2}}} \frac{\sqrt{E\left(p_{*}\right) E\left(p^{\prime}\right) E\left(p_{*}^{\prime}\right)}}{\left(g n_{c}\right)^{\frac{3}{2}}}+\sqrt{\frac{g n_{c}}{2}}\left(\sqrt{\frac{E\left(p_{*}^{\prime}\right)}{E\left(p_{*}\right) E\left(p^{\prime}\right)}}+\sqrt{\frac{E\left(p^{\prime}\right)}{E\left(p_{*}\right) E\left(p_{*}^{\prime}\right)}}-\sqrt{\frac{E\left(p_{*}\right)}{E\left(p^{\prime}\right) E\left(p_{*}^{\prime}\right)}}\right) .
$$

And so recalling that $E\left(p_{*}\right)=E\left(p^{\prime}\right)+E\left(p_{*}^{\prime}\right)$, we obtain

$$
A=\frac{1}{2^{\frac{5}{2}}} \frac{\sqrt{E\left(p_{*}\right) E\left(p^{\prime}\right) E\left(p_{*}^{\prime}\right)}}{\left(g n_{c}\right)^{\frac{3}{2}}} .
$$

With this $A$, the collision operator becomes

$$
\begin{array}{r}
C\left(f, n_{c}\right)(p)=\int \chi \frac{E\left(p_{1}\right) E\left(p_{2}\right) E\left(p_{3}\right)}{g^{3} n_{c}^{2}} \delta\left(p_{1}-p_{2}-p_{3}\right) \delta\left(E_{1}-E_{2}-E_{3}\right)\left[\delta\left(p-p_{1}\right)\right. \\
\left.-\delta\left(p-p_{2}\right)-\delta\left(p-p_{3}\right)\right]\left(\left(1+f_{1}\right) f_{2} f_{3}-f_{1}\left(1+f_{2}\right)\left(1+f_{3}\right)\right) d p_{1} d p_{2} d p_{3}
\end{array}
$$

where $\chi$ denotes the truncation for $\left|p_{i}\right| \leq \lambda, 1 \leq i \leq 3$. The choice of the positive constant $\lambda$ will be discussed below. 
The opposite limit of intermediate temperatures compared to $T_{B E C}$, and where all momenta $\left|p_{i}\right|>>$ $p_{0}$, is considered in $[\mathrm{E}, \mathrm{ZNG}]$ with the dominant excitation of Hartree-Fock single particle type. Expanding the square root definition of $E$ in (2.4), we may approximate $E_{p}$ by $\frac{p^{2}}{2 m}+g n_{c}$ leading to a collision operator of the type

$$
\begin{aligned}
& C\left(f, n_{c}\right)=k n_{c} \int_{\mathbb{R}^{3} \times \mathbb{R}^{3} \times \mathbb{R}^{3}} \chi \delta\left(p_{1}-p_{2}-p_{3}\right) \delta\left(E_{1}-E_{2}-E_{3}\right)\left[\delta\left(p-p_{1}\right)\right. \\
& \left.-\delta\left(p-p_{2}\right)-\delta\left(p-p_{3}\right)\right]\left(\left(1+f_{1}\right) f_{2} f_{3}-f_{1}\left(1+f_{2}\right)\left(1+f_{3}\right)\right) d p_{1} d p_{2} d p_{3}
\end{aligned}
$$

(for the 'partial local equilibrium regime' of [ZNG], see also [ITG], with only collisions between excited particles and the condensate). Here $\chi$ is the characteristic function of the set of $\left(p, p_{1}, p_{2}, p_{3}\right)$ with $|p|,\left|p_{1}\right|,\left|p_{2}\right|,\left|p_{3}\right| \geq \alpha$ for a given positive constant $\alpha$.

In the general case, the collision operator is

$$
\begin{array}{r}
C\left(f, n_{c}\right)(p)=n_{c} \int|A|^{2} \delta\left(p_{1}-p_{2}-p_{3}\right) \delta\left(E_{1}-E_{2}-E_{3}\right)\left[\delta\left(p-p_{1}\right)\right. \\
\left.-\delta\left(p-p_{2}\right)-\delta\left(p-p_{3}\right)\right]\left(\left(1+f_{1}\right) f_{2} f_{3}-f_{1}\left(1+f_{2}\right)\left(1+f_{3}\right)\right) d p_{1} d p_{2} d p_{3},
\end{array}
$$

with the excitation energy $E$ defined by

$$
E(p)=|p| \sqrt{\frac{p^{2}}{4 m^{2}}+\frac{g n_{c}}{m}} .
$$

As follows from the definitions of $A$ and $E(p)$ above, the kernel $|A|^{2}$ is bounded by a multiple of

$$
|\bar{A}|^{2}:=\left(\frac{\left|p_{1}\right|}{\sqrt{n_{c}}} \wedge 1\right)\left(\frac{\left|p_{2}\right|}{\sqrt{n_{c}}} \wedge 1\right)\left(\frac{\left|p_{3}\right|}{\sqrt{n_{c}}} \wedge 1\right)
$$

in the physically interesting cases when asymptotically all $\left|p_{i}\right|<<p_{0}$, all $\left|p_{i}\right|>>p_{0}$, or one $\left|p_{i}\right|<<$ $p_{0}$ and the others $>>p_{0}$. The three cases are relevant for low respectively intermediate temperatures compared to $T_{B E C}$, and (the third case) for the collision of low temperature phonons with high temperature excitations (atoms). The asymptotic situation of two $\left|p_{i}\right|<<p_{0}$ and one $p_{i}>>p_{0}$ (with unbounded $A$ ) is excluded by the energy condition.

In the main part of this paper we shall use $|\bar{A}|^{2}$ as the kernel in the collision operator and prove the following result.

Theorem 2.1 Let $n_{c i}>0$ and $f_{i}(p)=f_{i}(|p|) \in L^{1}$ be given with $f_{i}$ nonnegative and $f_{i}(p)|p|^{2+\gamma} \in$ $L^{1}$ for some $\gamma>0$. For the collision operator (2.7) with the transition probability kernel $|\bar{A}|^{2}$, there exists a nonnegative solution $\left(f, n_{c}\right) \in C^{1}\left([0, \infty) ; L_{+}^{1}\right) \times C^{1}([0, \infty))$ to the initial value problem (2.1-3). The condensate density $n_{c}$ is locally bounded away from zero for $t>0$. The excitation density $f$ has energy locally bounded in time. Total mass $M_{0}=n_{c i}+\int f_{i}(p) d p$ is conserved, and the moment $\int|p|^{2+\gamma} f d p$ is locally bounded in time.

In the low temperature case with the collision operator taken as (2.5), if the mathematical condition corresponding to the physics requirement $|p|<<p_{0}$ is taken as $|p| \leq p_{0}^{2}:=\lambda$, the proof of Theorem 2.1 simplifies. It holds that

Theorem 2.2 Let $n_{c i}>0$ and $f_{i}(p)=f_{i}(|p|) \in L^{1}$ be given with $f_{i}$ nonnegative. There exists a nonnegative solution $\left(f, n_{c}\right) \in C^{1}\left([0, \infty) ; L_{+}^{1}\right) \times C^{1}([0, \infty))$ to the initial value problem (2.1-3) for the collision operator (2.5). The condensate density $n_{c}$ is locally bounded away from zero for $t>0$. The excitation density $f$ has energy bounded globally in time. Total mass $M_{0}=n_{c i}+\int f_{i}(p) d p$ is conserved. 
An existence result was obtained in $[\mathrm{N}]$ for (2.1-3) in the intermediate temperature case, with the collision operator (2.6) without the cut-off function $\chi$, and considering the excitation density $f$ in measure sense. For (2.6) with the cut-off function $\chi$ included, existence also holds in the present $L^{1}$-setting.

Theorem 2.3 Let $n_{c i}>0$ and $f_{i}(p)=f_{i}(|p|) \in L^{1}$ be given with $f_{i}$ nonnegative and $f_{i}(p)|p|^{2+\gamma} \in$ $L^{1}$ for some $\gamma>0$. There exists a nonnegative solution $\left(f, n_{c}\right) \in C^{1}\left([0, \infty) ; L_{+}^{1}\right) \times C^{1}([0, \infty))$ to the initial value problem (2.1-3) for the collision operator (2.6). The condensate density $n_{c}$ is locally bounded away from zero for $t>0$. Total mass $M_{0}=n_{c i}+\int f_{i}(p) d p$ is conserved together with

the integral $\int \frac{p^{2}}{2 m} f_{i}(p) d p+\frac{1}{2} g n_{c i} \int f_{i}(p) d p+g M_{0}\left(\int f_{i}(p) d p+\frac{1}{2} n_{c}\right)$ of energy type. The moment $\int|p|^{2+\gamma} f d p$ is locally bounded in time.

Also the recent paper [S2] considers the spatially homogeneous and isotropic kinetic regime of weakly interacting bosons with s-wave scattering. It has a focus on post-nucleation self-similar solutions. Another recent paper, [EPV], studies linearized space homogeneous kinetic problems in settings related to but not identical to the ones discussed here, and with a focus on large time behaviour.

\section{Proof of the main theorem.}

The collision operator in the general case is

$$
\begin{array}{r}
C\left(f, n_{c}\right)(p)=n_{c} \int|\bar{A}|^{2} \delta\left(p_{1}-p_{2}-p_{3}\right) \delta\left(E_{1}-E_{2}-E_{3}\right)\left[\delta\left(p-p_{1}\right)\right. \\
\left.-\delta\left(p-p_{2}\right)-\delta\left(p-p_{3}\right)\right]\left(\left(1+f_{1}\right) f_{2} f_{3}-f_{1}\left(1+f_{2}\right)\left(1+f_{3}\right)\right) d p_{1} d p_{2} d p_{3},
\end{array}
$$

with the kernel

$$
|\bar{A}|^{2}:=\left(\frac{\left|p_{1}\right|}{\sqrt{n_{c}}} \wedge 1\right)\left(\frac{\left|p_{2}\right|}{\sqrt{n_{c}}} \wedge 1\right)\left(\frac{\left|p_{3}\right|}{\sqrt{n_{c}}} \wedge 1\right)
$$

and the excitation energy $E$ defined by

$$
E\left(p, n_{c}\right)=E(p)=E_{p}=|p| \sqrt{\frac{p^{2}}{4 m^{2}}+\frac{g n_{c}}{m}} .
$$

The constants $m$ and $g$ are taken as $\frac{1}{2}$ in the rest of this section. Adding (2.2) and (2.1) integrated with respect to $p$, it follows that $n_{c}(t)+\int f(t,|p|) d p=M_{0}$, i.e. total mass is conserved. It holds that

$$
\begin{array}{r}
\int \varphi(p) C\left(f, n_{c}\right)(p) d p=n_{c} \int\left|\bar{A}^{2}\right|\left(\varphi\left(p_{1}\right)-\varphi\left(p_{2}\right)-\varphi\left(p_{3}\right)\right) \delta\left(p_{1}-p_{2}-p_{3}\right) \\
\delta\left(E\left(p_{1}\right)-E\left(p_{2}\right)-E\left(p_{3}\right)\right)\left(f_{2} f_{3}-f_{1}\left(1+f_{2}+f_{3}\right)\right) d p_{1} d p_{2} d p_{3} .
\end{array}
$$

The energy (resp. the condensate density) is bounded from above (resp. from below) locally in time as follows.

Lemma 3.1 Let the initial data $\left(f_{i}, n_{c i}\right)$ satisfy $0<n_{c i}<M_{0}$ and $n_{c i}+\int f_{i}(|p|) d p=M_{0}$. Then there is $T_{0}>0$ such that $n_{c}(t) \geq \frac{n_{c i}}{2}$ and $\int E\left(p, n_{c}\right) f(t, p) d p$ is bounded from above on $\left[0, T_{0}\right]$ for any nonnegative solution $\left(f, n_{c}\right)$ to (2.1-3). 


\section{Proof of Lemma 3.1}

It follows from (2.2) and (3.1) that for any nonnegative solution $\left(f, n_{c}\right)$ to $(2.1-3)$,

$$
\begin{array}{r}
\left|\frac{n_{c}^{\prime}}{n_{c}}(t)\right| \leq \int\left(\frac{\left|p_{1}\right|}{\sqrt{n_{c}}} \wedge 1\right)\left(\frac{\left|p_{2}\right|}{\sqrt{n_{c}}} \wedge 1\right)\left(\frac{\left|p_{3}\right|}{\sqrt{n_{c}}} \wedge 1\right) \delta\left(p_{1}-p_{2}-p_{3}\right) \delta\left(E_{1}-E_{2}-E_{3}\right) \\
\left(f_{2} f_{3}+f_{1}\left(2 f_{2}+1\right)\right) d p_{1} d p_{2} d p_{3}=: X_{1}+X_{2}+X_{3} .
\end{array}
$$

Using spherical coordinates for $p_{2}$ and $p_{3}$, with axis directed by $p_{2}$ and azimuthal angle $\varphi_{3}$ for $p_{3}$, setting $|p|=r$, and then performing the change of variables $\varphi_{3} \rightarrow s=\cos \varphi_{3}$,

$$
X_{1} \leq 2 k \int_{0}^{\infty} r_{2}^{2}\left(\frac{r_{2}}{\sqrt{n_{c}}} \wedge 1\right) f\left(t, r_{2}\right) \int_{0}^{r_{2}} r_{3}^{2}\left(\frac{r_{3}}{\sqrt{n_{c}}} \wedge 1\right) f\left(t, r_{3}\right) Y_{1} d r_{3} d r_{2},
$$

where

$$
\begin{array}{r}
Y_{1}=\int_{-1}^{1} \delta\left(F_{1}(s)\right) d s, \\
F_{1}(s):=\sqrt{\left(r_{2}^{2}+r_{3}^{2}+2 r_{2} r_{3} s\right)^{2}+n_{c}\left(r_{2}^{2}+r_{3}^{2}+2 r_{2} r_{3} s\right)}-S_{1}, \\
S_{1}:=\sqrt{r_{2}^{4}+n_{c} r_{2}^{2}}+\sqrt{r_{3}^{4}+n_{c} r_{3}^{2}} .
\end{array}
$$

$F_{1}$ vanishes for a single value $s_{1}$ of $s$. Straightforward computations show that $\left|s_{1}\right| \leq 1$. Moreover,

$$
F_{1}^{\prime}(s)=r_{2} r_{3} \frac{2\left(r_{2}^{2}+r_{3}^{2}+2 r_{2} r_{3} s\right)+n_{c}}{\sqrt{\left(r_{2}^{2}+r_{3}^{2}+2 r_{2} r_{3} s\right)^{2}+n_{c}\left(r_{2}^{2}+r_{3}^{2}+2 r_{2} r_{3} s\right)}} \geq r_{2} r_{3} \frac{\sqrt{\left(r_{2}^{2}+r_{3}^{2}+2 r_{2} r_{3} s\right)+n_{c}}}{\sqrt{r_{2}^{2}+r_{3}^{2}+2 r_{2} r_{3} s}} .
$$

And so,

$$
Y_{1} \leq \frac{1}{r_{2} r_{3}}
$$

Hence,

$$
\begin{array}{r}
X_{1} \leq 2 k \int_{0}^{\infty} r_{2}\left(\frac{r_{2}}{\sqrt{n_{c}}} \wedge 1\right) f\left(t, r_{2}\right) \int_{0}^{r_{2}} r_{3}\left(\frac{r_{3}}{\sqrt{n_{c}}} \wedge 1\right) f\left(t, r_{3}\right) d r_{3} d r_{2} \\
\leq \frac{2 k}{n_{c}}\left(\int f(t, p) d p\right)^{2} .
\end{array}
$$

Similarly,

$$
X_{2} \leq 2 k \int_{0}^{\infty} r_{1}^{2}\left(\frac{r_{1}}{\sqrt{n_{c}}} \wedge 1\right) f\left(t, r_{1}\right) \int_{0}^{r_{1}} r_{2}^{2}\left(\frac{r_{2}}{\sqrt{n_{c}}} \wedge 1\right) f\left(t, r_{2}\right) Y_{2} d r_{1} d r_{2},
$$

where

$$
\begin{array}{r}
Y_{2}=\int_{-1}^{1} \delta\left(F_{2}(s)\right) d s, \\
F_{2}(s):=\sqrt{\left(r_{1}^{2}+r_{2}^{2}+2 r_{1} r_{2} s\right)^{2}+n_{c}\left(r_{1}^{2}+r_{2}^{2}+2 r_{1} r_{2} s\right)}-S_{2}, \\
S_{2}:=\sqrt{r_{1}^{4}+n_{c} r_{1}^{2}}-\sqrt{r_{2}^{4}+n_{c} r_{2}^{2}} .
\end{array}
$$

$F_{2}$ vanishes for a single value $s_{2}$ of $s$. Straightforward computations show that $\left|s_{2}\right| \leq 1$. Moreover,

$$
F_{2}^{\prime}(s)=r_{1} r_{2} \frac{2\left(r_{1}^{2}+r_{2}^{2}+2 r_{1} r_{2} s\right)+n_{c}}{\sqrt{\left(r_{1}^{2}+r_{2}^{2}+2 r_{1} r_{2} s\right)^{2}+n_{c}\left(r_{1}^{2}+r_{2}^{2}+2 r_{1} r_{2} s\right)}} \geq r_{1} r_{2} \frac{\sqrt{\left(r_{1}^{2}+r_{2}^{2}+2 r_{1} r_{2} s\right)+n_{c}}}{\sqrt{r_{2}^{1}+r_{3}^{2}+2 r_{1} r_{2} s}}
$$


And so,

$$
\begin{array}{r}
Y_{2} \leq \frac{1}{r_{1} r_{2}}, \\
X_{2} \leq \frac{2 k}{n_{c}}\left(\int f(t, p) d p\right)^{2} .
\end{array}
$$

Finally,

$$
\begin{array}{r}
X_{3} \leq k \int r_{1}\left(\frac{r_{1}}{\sqrt{n_{c}}} \wedge 1\right) f\left(t, r_{1}\right) \int_{0}^{r_{1}} r_{2}\left(\frac{r_{2}}{\sqrt{n_{c}}} \wedge 1\right) d r_{2} d r_{1} \\
=\frac{k}{\sqrt{n_{c}}} \int_{0}^{\sqrt{n_{c}}} r_{1}^{2} f\left(t, r_{1}\right) \int_{0}^{r_{1}} \frac{r_{2}^{2}}{\sqrt{n_{c}}} d r_{2} d r_{1}+k \int_{\sqrt{n_{c}}}^{+\infty} r_{1} f\left(t, r_{1}\right)\left(\int_{0}^{\sqrt{n_{c}}} \frac{r_{2}^{2}}{\sqrt{n_{c}}} d r_{2}+\int_{\sqrt{n_{c}}}^{r_{1}} r_{2} d r_{2}\right) d r_{1} \\
\leq \frac{k}{n_{c}} \int_{0}^{\sqrt{n_{c}}} r_{1}^{5} f\left(t, r_{1}\right) d r_{1}+\frac{k}{2} \int_{\sqrt{n_{c}}}^{+\infty} r_{1}^{3} f\left(t, r_{1}\right) d r_{1} \\
\leq k \sqrt{n_{c}} \int f(t, p) d p+\frac{k}{2 \sqrt{n_{c}}} \int p^{2} f(t, p) d p \leq M_{0} k \sqrt{n_{c}}+\frac{k}{2 \sqrt{n_{c}}} \int p^{2} f(t, p) d p .
\end{array}
$$

And so,

$$
\left|n_{c}^{\prime}(t)\right| \leq k\left(4 M_{0}^{2}+M_{0} n_{c}^{\frac{3}{2}}+\sqrt{n_{c}} \int p^{2} f(t, p) d p\right) .
$$

Denote by $G(t, n)=\int E(p, n) f(t, p) d p$. Then

$$
\frac{\partial G}{\partial n}=\int \frac{|p|}{2 \sqrt{p^{2}+n}} f(t, p) d p \in\left[0, \frac{M_{0}}{2}\right] .
$$

Hence,

$$
G\left(t, n_{c}(t)\right) \leq G\left(t, n_{c i}\right)+M_{0}^{2} .
$$

Moreover,

$$
\begin{array}{r}
\frac{d}{d t} G\left(t, n_{c i}\right)=\int|p| \sqrt{p^{2}+n_{c i}} C\left(f, n_{c}\right)(t, p) d p \\
=n_{c} \int\left(\left|\frac{\left|p_{1}\right|}{\sqrt{n_{c}}}\right| \wedge 1\right)\left(\left|\frac{\left|p_{2}\right|}{\sqrt{n_{c}}}\right| \wedge 1\right)\left(\left|\frac{\left|p_{3}\right|}{\sqrt{n_{c}}}\right| \wedge 1\right) \\
\left(\left|p_{1}\right| \sqrt{p_{1}^{2}+n_{c i}}-\left|p_{2}\right| \sqrt{p_{2}^{2}+n_{c i}}-\left|p_{3}\right| \sqrt{p_{3}^{2}+n_{c i}}\right) \\
\delta\left(p_{1}-p_{2}-p_{3}\right) \delta\left(\left|p_{1}\right| \sqrt{p_{1}^{2}+n_{c}(t)}-\left|p_{2}\right| \sqrt{p_{2}^{2}+n_{c}(t)}-\left|p_{3}\right| \sqrt{p_{3}^{2}+n_{c}(t)}\right) \\
\left(f_{2} f_{3}-f_{1}\left(1+f_{2}+f_{3}\right) d p_{1} d p_{2} d p_{3}\right. \\
=n_{c} \int|\bar{A}|^{2}\left(\left|p_{1}\right|\left(\sqrt{p_{1}^{2}+n_{c i}}-\sqrt{p_{1}^{2}+n_{c}(t)}\right)-\left|p_{2}\right|\left(\sqrt{p_{2}^{2}+n_{c i}}-\sqrt{p_{2}^{2}+n_{c}(t)}\right)\right. \\
-\left|p_{3}\right|\left(\sqrt{p_{3}^{2}+n_{c i}}-\sqrt{\left.p_{3}^{2}+n_{c}(t)\right)}\right) \\
\delta\left(p_{1}-p_{2}-p_{3}\right) \delta\left(\left|p_{1}\right| \sqrt{p_{1}^{2}+n_{c}(t)}-\left|p_{2}\right| \sqrt{p_{2}^{2}+n_{c}(t)}-\left|p_{3}\right| \sqrt{p_{3}^{2}+n_{c}(t)}\right) \\
\left(f_{2} f_{3}-f_{1}\left(1+f_{2}+f_{3}\right) d p_{1} d p_{2} d p_{3} .\right.
\end{array}
$$

It follows from

$$
|p|\left|\sqrt{p^{2}+n_{c i}}-\sqrt{p^{2}+n_{c}(t)}\right| \leq\left|n_{c i}-n_{c}(t)\right| \leq M_{0}, \quad p \in \mathbb{R}^{3},
$$


and similar computations as in the control of $X_{1}, X_{2}$ and $X_{3}$ above, that

$$
\begin{array}{r}
\left|\frac{d}{d t} G\left(t, n_{c i}\right)\right| \leq 2 k M_{0} n_{c}(t)\left(\frac{4 M_{0}^{2}}{n_{c}(t)}+2 M_{0} \sqrt{n_{c}(t)}+\frac{1}{\sqrt{n_{c}}} \int p^{2} f(t, p) d p\right) \\
\leq 2 k M_{0} n_{c}(t)\left(\frac{4 M_{0}^{2}}{n_{c}(t)}+2 M_{0} \sqrt{n_{c}(t)}+\frac{G\left(t, n_{c i}\right)}{\sqrt{n_{c}}}\right) .
\end{array}
$$

And so,

$$
G\left(t, n_{c}(t)\right) \leq M_{0}^{2}+G\left(0, n_{c i}\right) \exp \left(2 M_{0}^{\frac{3}{2}} t\right)+k\left(8 M_{0}^{3}+2 M_{0}^{4}+2 M_{0}^{\frac{5}{2}}\right)\left(\exp \left(2 M_{0}^{\frac{3}{2}} t\right)-1\right) .
$$

The lemma follows.

If the solution exists on $[0, T$, then it follows from a refinement of (3.2) in the proof of Lemma 3.1 that $\inf _{[0, T[} n_{c}(t)>0$. For a contradiction assume that $\liminf _{t \rightarrow T} n_{c}(t)=0$, which implies $\lim _{t \rightarrow T} n_{c}(t)=0$. By (3.4), the energy is uniformly bounded on $[0, T[$. By $(2.2)$ and (3.1)

$$
\begin{array}{r}
\frac{n_{c}^{\prime}}{n_{c}}(t)=\int\left(\frac{\left|p_{1}\right|}{\sqrt{n_{c}}} \wedge 1\right)\left(\frac{\left|p_{2}\right|}{\sqrt{n_{c}}} \wedge 1\right)\left(\frac{\left|p_{3}\right|}{\sqrt{n_{c}}} \wedge 1\right) \delta\left(p_{1}-p_{2}-p_{3}\right) \delta\left(E_{1}-E_{2}-E_{3}\right) \\
\left(f_{2} f_{3}-f_{1}\left(2 f_{2}+1\right)\right) d p_{1} d p_{2} d p_{3} .
\end{array}
$$

This gives

$$
\begin{aligned}
& \frac{n_{c}^{\prime}}{n_{c}}(t)=16 \pi^{2} \int_{0}^{\infty} y^{2}\left(\frac{y}{\sqrt{n_{c}}} \wedge 1\right) f(t, y) \int_{0}^{y} z^{2}\left(\frac{z}{\sqrt{n_{c}}} \wedge 1\right) f(t, z) \\
& \left(\frac{1}{\left|F_{1}^{\prime}\left(s_{1}\right)\right|}\left(\sqrt{\frac{y^{2}+z^{2}+2 y z s_{1}}{n_{c}}} \wedge 1\right)-\frac{1}{\left|F_{2}^{\prime}\left(s_{2}\right)\right|}\left(\sqrt{\frac{y^{2}+z^{2}+2 y z s_{2}}{n_{c}}} \wedge 1\right)\right) d z d y \\
& -\int\left(\frac{\left|p_{1}\right|}{\sqrt{n_{c}}} \wedge 1\right)\left(\frac{\left|p_{2}\right|}{\sqrt{n_{c}}} \wedge 1\right)\left(\frac{\left|p_{3}\right|}{\sqrt{n_{c}}} \wedge 1\right) \delta\left(p_{1}-p_{2}-p_{3}\right) \delta\left(E_{1}-E_{2}-E_{3}\right) f_{1} d p_{1} d p_{2} d p_{3} \text {. }
\end{aligned}
$$

But $s_{1} \geq s_{2}$, since

$2\left(y^{2}+z^{2}+2 y z s_{1}\right)=\sqrt{n_{c}^{2}+4(E(y)+E(z))^{2}}-n_{c} \geq \sqrt{n_{c}^{2}+4(E(y)-E(z))^{2}}-n_{c}=2\left(y^{2}+z^{2}+2 y z s_{2}\right)$.

Moreover, $F_{1}^{\prime}=F_{2}^{\prime}$ is a non-increasing function. And so, for $(y, z) \neq(0,0)$,

$$
\frac{1}{\left|F_{1}^{\prime}\left(s_{1}\right)\right|}\left(\sqrt{\frac{y^{2}+z^{2}+2 y z s_{1}}{n_{c}}} \wedge 1\right)-\frac{1}{\left|F_{2}^{\prime}\left(s_{2}\right)\right|}\left(\sqrt{\frac{y^{2}+z^{2}+2 y z s_{2}}{n_{c}}} \wedge 1\right)
$$

is positive. Thus, the first term in the r.h.s. of (3.5) is continuous and positive for $t \in[0, T$. Hence for some $k>0$,

$$
n_{c}^{\prime}(t) \geq k-2 M_{0} n_{c}^{\frac{3}{2}}-\sqrt{n_{c}} \int p^{2} f(t, p) d p
$$

for $t<T$, with the integral $\int p^{2} f(t, p) d p$ bounded. This contradicts $\lim _{t \rightarrow T} n_{c}(t)=0$.

\section{Lemma 3.2}

$$
\left|\int C\left(f, n_{c}\right)(p) d p\right| \leq k\left(\int f(p) d p\right)^{2}+k \sqrt{n_{c}} \int p^{2} f(p) d p
$$


Proof of Lemma 3.2.

$$
\begin{array}{r}
\left|\int C\left(f, n_{c}\right)(p) d p\right| \leq n_{c} \int\left(\frac{\left|p_{1}\right|}{\sqrt{n_{c}}} \wedge 1\right)\left(\frac{\left|p_{2}\right|}{\sqrt{n_{c}}} \wedge 1\right)\left(\frac{\left|p_{3}\right|}{\sqrt{n_{c}}} \wedge 1\right) \\
\delta\left(p_{1}-p_{2}-p_{3}\right) \delta\left(E\left(p_{1}\right)-E\left(p_{2}\right)-E\left(p_{3}\right)\right)\left(f_{2} f_{3}+f_{1}\left(1+f_{2}+f_{3}\right)\right) d p_{1} d p_{2} d p_{3} .
\end{array}
$$

To control the $f_{2} f_{3}$ term of (3.6), use spherical coordinates for $p_{2}$ and $p_{3}$ with the axis for $p_{3}$ directed by $p_{2}$ and denote by $\varphi_{3}$ the azimuthal angle related to $p_{3}$. Then

$$
\begin{array}{r}
\int\left(\frac{\left|p_{1}\right|}{\sqrt{n_{c}}} \wedge 1\right)\left(\frac{\left|p_{2}\right|}{\sqrt{n_{c}}} \wedge 1\right)\left(\frac{\left|p_{3}\right|}{\sqrt{n_{c}}} \wedge 1\right) \delta\left(p_{1}-p_{2}-p_{3}\right) \delta\left(E\left(p_{1}\right)-E\left(p_{2}\right)-E\left(p_{3}\right)\right) f_{2} f_{3} d p_{1} d p_{2} d p_{3} \leq \\
k \int r_{2}^{2}\left(\frac{r_{2}}{\sqrt{n_{c}}} \wedge 1\right) f_{2} \int r_{3}^{2}\left(\frac{r_{3}}{\sqrt{n_{c}}} \wedge 1\right) f_{3} Y_{1} d r_{2} d r_{3}
\end{array}
$$

where

$$
Y_{1}=\int_{-1}^{1} \delta\left(\sqrt{\left(r_{2}^{2}+r_{3}^{2}+2 r_{2} r_{3} s\right)^{2}+n\left(r_{2}^{2}+r_{3}^{2}+2 r_{2} r_{3} s\right)}-E_{2}-E_{3}\right) d s \leq \frac{1}{r_{2} r_{3}} .
$$

And so,

$$
\begin{array}{r}
\left.n_{c} \int\left(\frac{\left|p_{1}\right|}{\sqrt{n_{c}}} \wedge 1\right)\left(\frac{\left|p_{2}\right|}{\sqrt{n_{c}}} \wedge 1\right)\left(\frac{\left|p_{3}\right|}{\sqrt{n_{c}}} \wedge 1\right) \delta\left(p_{1}-p_{2}-p_{3}\right)\right) \delta\left(E\left(p_{1}\right)-E\left(p_{2}\right)-E\left(p_{3}\right)\right) f\left(p_{2}\right) f\left(p_{3}\right) d p_{1} d p_{2} d p_{3} \\
\leq k\left(\int f(p) d p\right)^{2} .
\end{array}
$$

In the same way the other quadratic terms of (3.6) can be bounded by $k\left(\int f(p) d p\right)^{2}$. Finally,

$$
\begin{aligned}
n_{c} \int\left(\frac{\left|p_{1}\right|}{\sqrt{n_{c}}} \wedge 1\right)\left(\frac{\left|p_{2}\right|}{\sqrt{n_{c}}} \wedge 1\right)\left(\frac{\left|p_{3}\right|}{\sqrt{n_{c}}}\right. & \wedge 1) \delta\left(p_{1}-p_{2}-p_{3}\right) \delta\left(E\left(p_{1}\right)-E\left(p_{2}\right)-E\left(p_{3}\right)\right) f_{1} d p_{1} d p_{2} d p_{3} \\
& \leq k n_{c} \int r_{1} f_{1} \int_{0}^{r_{1}}\left(\frac{r_{2}}{\sqrt{n_{c}}} \wedge 1\right) r_{2} d r_{2} d r_{1} \leq k \sqrt{n_{c}} \int p^{2} f(p) d p .
\end{aligned}
$$

Lemma 3.3 Given $0<n_{*}<M_{0}$, there is a constant $k$ such that for any $n \in\left[n_{*}, M_{0}\right]$ and isotropic functions $(f, g) \in L_{+}^{1}\left(\mathbb{R}^{3}\right) \times L_{+}^{1}\left(\mathbb{R}^{3}\right)$ with $L^{1}$ norm bounded by $M_{0}$,

$$
\int|(C(f, n)-C(g, n))(p)| d p \leq k \int\left(1+\sqrt{n} p^{2}\right)|(f-g)(p)| d p
$$

with $k$ independent of $n, f, g$.

Proof of Lemma 3.3.

Denote by $\mu(f)=\left(f_{2} f_{3}-f_{1}\left(1+f_{2}+f_{3}\right)\right)$. Then by computations similar to those used in the proof of Lemma 3.2,

$$
\begin{array}{r}
\int|(C(f, n)-C(g, n))(p)| d p \\
\leq k n \int\left|p_{1}\right| \wedge 1\left|p_{2}\right| \wedge 1\left|p_{3}\right| \wedge 1 \delta\left(p_{1}-p_{2}-p_{3}\right) \delta\left(E_{1}-E_{2}-E_{3}\right)|\mu(f)-\mu(g)| d p_{1} d p_{2} d p_{3} \\
\leq k\left(\int f d p+\int g d p\right) \int|f(p)-g(p)| d p+k \sqrt{n} \int p^{2}|f(p)-g(p)| d p .
\end{array}
$$


Lemma 3.4 For any $\gamma \in[0,1]$,

$$
\int|p|^{2+2 \gamma} f(t, p) d p \leq \int|p|^{2+2 \gamma} f_{i}(p) d p+M_{0} t \sup _{s \leq t}\left(\int\left(1+p^{2}\right) f(s, p) d p\right)^{2}
$$

Proof of Lemma 3.4.

Let $\gamma \in[0,1]$ be fixed. Multiplying the equation satisfied by $f$ by $|p|^{2+2 \gamma}$ and integrating it on $(0, t) \times \mathbb{R}^{3}$ leads to

$$
\begin{array}{r}
\int|p|^{2+2 \gamma} f(t, p) d p+\int_{0}^{t} n_{c}(s) \int|\bar{A}|^{2}\left(\left|p_{1}\right|^{2+2 \gamma}-\left|p_{2}\right|^{2+2 \gamma}\right. \\
\left.-\left|p_{3}\right|^{2+2 \gamma}\right) f_{1} \delta\left(p_{1}=p_{2}+p_{3}\right) \delta\left(E_{1}=E_{2}+E_{3}\right) d p_{1} d p_{2} d p_{3} d s \\
=\int|p|^{2+2 \gamma} f_{i}(p) d p+\int_{0}^{t} n_{c}(s) \int|\bar{A}|^{2}\left(\left|p_{1}\right|^{2+2 \gamma}-\left|p_{2}\right|^{2+2 \gamma}-\left|p_{3}\right|^{2+2 \gamma}\right)\left(f_{2} f_{3}\right. \\
\left.-f_{1}\left(f_{2}+f_{3}\right)\right) \delta\left(p_{1}=p_{2}+p_{3}\right) \delta\left(E_{1}=E_{2}+E_{3}\right) d p_{1} d p_{2} d p_{3} d s .
\end{array}
$$

It is sufficient to prove that there is a positive constant $\tilde{K}$ such that

$$
0 \leq r_{1}^{2+2 \gamma}-r_{2}^{2+2 \gamma}-r_{3}^{2+2 \gamma} \leq \tilde{K}\left(1+r_{2}^{2}\right)\left(1+r_{3}^{2}\right),
$$

when $E_{1}=E_{2}+E_{3}$. Indeed, the second term in the left member of (3.8) will then be nonnegative, whereas the second term in the right member will be bounded from above by $M_{0} t \sup _{s \leq t}\left(\int\left(1+p^{2}\right) f(s, p) d p\right)^{2}$. Since

$$
r_{i}^{2}=\frac{\sqrt{n^{2}+4 E_{i}^{2}}-n}{2}, \quad 1 \leq i \leq 3,
$$

(3.8) holds if there is a positive constant $K$ such that

$$
\begin{array}{r}
0 \leq\left(\sqrt{n^{2}+4\left(E_{2}+E_{3}\right)^{2}}-n\right)^{1+\gamma}-\left(\sqrt{n^{2}+4 E_{2}^{2}}-n\right)^{1+\gamma}-\left(\sqrt{n^{2}+4 E_{3}^{2}}-n\right)^{1+\gamma} \\
\leq K\left(\sqrt{n^{2}+4 E_{2}^{2}}-n+2\right)\left(\sqrt{n^{2}+4 E_{3}^{2}}-n+2\right), \quad\left(E_{2}, E_{3}\right) \in\left(\mathbb{R}_{+}\right)^{2} .
\end{array}
$$

To prove the left inequality of (3.9), consider the function

$$
g\left(x, E_{3}\right):=\left(\sqrt{n^{2}+4\left(x+E_{3}\right)^{2}}-n\right)^{1+\gamma}-\left(\sqrt{n^{2}+4 x^{2}}-n\right)^{1+\gamma}-\left(\sqrt{n^{2}+4 E_{3}^{2}}-n\right)^{1+\gamma}, \quad x \geq 0 .
$$

Then,

$$
\frac{\sqrt{n^{2}+4\left(x+E_{3}\right)^{2}} \sqrt{n^{2}+4 x^{2}}}{4(1+\gamma)} \frac{\partial g}{\partial x}\left(x, E_{3}\right)=: C,
$$

where

$$
C=\left(x+E_{3}\right) \sqrt{n^{2}+4 x^{2}}\left(\sqrt{n^{2}+4\left(x+E_{3}\right)^{2}}-n\right)^{\gamma}-x \sqrt{n^{2}+4\left(x+E_{3}\right)^{2}}\left(\sqrt{n^{2}+4 x^{2}}-n\right)^{\gamma}
$$

is nonnegative if

$$
\frac{x+E_{3}}{x} \sqrt{\frac{n^{2}+4 x^{2}}{n^{2}+4\left(x+E_{3}\right)^{2}}} \geq 1,
$$


which is true, since

$$
\left(\frac{x+E_{3}}{x}\right)^{2} \geq \frac{n^{2}+4\left(x+E_{3}\right)^{2}}{n^{2}+4 x^{2}}
$$

is equivalent to

$$
2 n^{2} E_{3} x+n^{2} E_{3}^{2} \geq 0 .
$$

And so, the function $g$ is non-decreasing in $x$. It follows from $g\left(0, E_{3}\right)=0$ that $g$ is non-negative. For the right inequality of (3.9), it is by symmetry enough to consider $E_{3} \leq x$. The inequality is obtained by proving that $g$ is bounded from above by a multiple of the function $h$ defined by

$$
h\left(x, E_{3}\right):=\left(\sqrt{n^{2}+4 x^{2}}+2-n\right)\left(\sqrt{n^{2}+4 E_{3}^{2}}+2-n\right), \quad x \geq 0 .
$$

For $h$ we notice that $\left(\sqrt{n^{2}+4 y^{2}}-n+2\right) \geq 2(\geq y)$ for $y \leq n(y \geq n)$. It follows that $h\left(x, E_{3}\right) \geq 4$ for $x, E_{3} \leq n, h\left(x, E_{3}\right) \geq 2 \cdot x$ for $x \geq n, E_{3} \leq n$, and $h\left(x, E_{3}\right) \geq x \cdot E_{3}$ for $x, E_{3} \geq n$.

For $x, E_{3} \leq n, g\left(x, E_{3}\right)$ is positive and bounded from above by some constant $c_{1}$. We thus require $K \geq \frac{c_{1}}{4}$. For $x, E_{3} \geq n$ we start from

$$
\left(\sqrt{n^{2}+4 y^{2}}-n\right)^{1+\gamma}=(2 y)^{1+\gamma}\left(\sqrt{\frac{n^{2}}{4 y^{2}}+1}-\frac{n}{2 y}\right)^{1+\gamma}=(2 y)^{1+\gamma}\left(1+\frac{n^{2}}{8 y^{2}}\left(\theta \frac{n^{2}}{4 y^{2}}+1\right)^{-\frac{1}{2}}-\frac{n}{2 y}\right)^{1+\gamma},
$$

which gives

$$
(2 y)^{1+\gamma}-(1+\gamma) \frac{15}{32} n y^{\gamma} \geq\left(\sqrt{n^{2}+4 y^{2}}-n\right)^{1+\gamma} \geq(2 y)^{1+\gamma}-(1+\gamma) 2^{\gamma} n y^{\gamma} .
$$

It follows that

$$
\begin{array}{r}
g\left(x, E_{3}\right) \leq 2^{1+\gamma}\left(\left(x+E_{3}\right)^{1+\gamma}-x^{1+\gamma}-E_{3}^{1+\gamma}\right)+(1+\gamma) 2^{\gamma} n\left(x^{\gamma}+E_{3}^{\gamma}\right) \\
=2^{1+\gamma}\left(x\left(\left(x+E_{3}\right)^{\gamma}-x^{\gamma}\right)+E_{3}\left(\left(x+E_{3}\right)^{\gamma}-E_{3}^{\gamma}\right)\right)+(1+\gamma) 2^{\gamma} n\left(x^{\gamma}+E_{3}^{\gamma}\right) \\
\leq 2^{2+2 \gamma} E_{3} x^{\gamma}+2^{2+\gamma} n x^{\gamma},
\end{array}
$$

which is bounded by a multiple of $h\left(x, E_{3}\right)$ in this domain. This also holds for $x \geq n, E_{3} \leq n$ with an analogous proof.

\section{Proof of Theorem 2.1.}

By Lemma 3.1, for any fixed initial data $f_{i}$, given $n_{c i}>0$, there is a positive time $T_{0}$ and $n_{*}>0$ such that any solution $n(t)$ of (2.1-3) is bounded from below by $n_{*}$ on $\left[0, T_{0}\right]$. Let $\mathcal{K}$ denote the closed and convex subset of $C\left(\left[0, T_{0}\right]\right)$ consisting of the functions $n$ in $C\left(\left[0, T_{0}\right]\right)$ such that $n(0)=n_{c i}$ and $\frac{n_{*}}{2} \leq n(t) \leq M_{0}, t \in\left[0, T_{0}\right]$. A local in time solution to equations (2.1-3) is found as a fixed point of the following map. Let a (large) truncation value $P$ be defined for the linear part of the collision operator. Let $\Phi$ be the map defined on $\mathcal{K}$ by $\Phi(n)=m$, where

$$
m(t)=M_{0}-\int f(t, p) d p, \quad t \in\left[0, T_{0}\right],
$$

and $f$ is the mild solution in $C\left(\left[0, \tau_{0}\right] ; L^{1}\right)$ for some $\tau_{0}$ defined below with $0<\tau_{0} \leq T_{0}$, to

$$
\begin{gathered}
\frac{\partial f}{\partial t}=C^{P}(f, n), \\
f(0, p)=f_{i}(p),
\end{gathered}
$$


with

$$
\begin{array}{r}
C^{P}(f, n)=n \int|\bar{A}|^{2} \delta\left(p_{1}-p_{2}-p_{3}\right) \delta\left(E_{1}-E_{2}-E_{3}\right)\left[\delta\left(p-p_{1}\right)\right. \\
\left.-\delta\left(p-p_{2}\right)-\delta\left(p-p_{3}\right)\right]\left(f_{2} f_{3}-f_{1}\left(f_{2}+f_{3}\right)\right) d p_{1} d p_{2} d p_{3} \\
-n \int \chi_{|p|<P}|\bar{A}|^{2} \delta\left(p_{1}-p_{2}-p_{3}\right) \delta\left(E_{1}-E_{2}-E_{3}\right)\left[\delta\left(p-p_{1}\right)-\delta\left(p-p_{2}\right)-\delta\left(p-p_{3}\right)\right] f_{1} d p_{1} d p_{2} d p_{3} .
\end{array}
$$

Here $\chi_{|p|<P}$ is the characteristic function of the set where $|p|<P$. We notice that the discussion after Lemma 3.1 also holds for (3.10). Writing the equation (3.10) in exponential form and estimating the solution from below by the term containing the initial value, it follows that the bound of $n$ from below, $n_{*}$, can be taken independent of $P$ because the first term to the right in (3.5) can in this way be bounded from below uniformly in $P$ on any time interval.

Given $n$, a mild solution $f$ for (3.10) can be constructed as the limit of the nonnegative sequence $\left(f_{j}\right)$, defined by $f_{0}=f_{i}$ and

$$
\begin{array}{r}
\frac{\partial f_{j+1}}{\partial t}+f_{j+1} C_{l}^{P}\left(f_{j}, n\right)=C_{g}^{P}\left(f_{j}, n\right) \\
f_{j+1}(0, p)=f_{i}(p) .
\end{array}
$$

The collision frequency is

$$
\begin{aligned}
C_{l}^{P}(f, n)= & n\left[2 \int|\bar{A}|^{2} \delta\left(p-p_{2}-p_{3}\right) \delta\left(E_{p}-E_{2}-E_{3}\right) f_{2} d p_{2} d p_{3}\right. \\
& +2 \int|\bar{A}|^{2} \delta\left(p_{1}-p_{2}-p\right) \delta\left(E_{1}-E_{2}-E_{p}\right) f_{2} d p_{1} d p_{2} \\
+ & \left.\int \chi_{|p|<P}|\bar{A}|^{2} \delta\left(p-p_{2}-p_{3}\right) \delta\left(E_{p}-E_{2}-E_{3}\right) d p_{2} d p_{3}\right]
\end{aligned}
$$

which preserves positivity together with the gain term $C_{g}^{P}(f, n)=C^{P}(f, n)+f C_{l}^{P}(f, n)$. For any nonnegative functions $f, g \in L^{1}$ and any $n \in\left[0, M_{0}\right]$, it holds that

$$
\int\left|\left(C^{P}(f, n)-C^{P}(g, n)\right)(p)\right| d p \leq k n\left(P^{2}+\int f(p) d p+\int g(p) d p\right) \int|(f-g)(p)| d p .
$$

For $\tau_{0}>0$ smaller than $\frac{c}{\int f_{i}(p) d p+P^{2}}$, where $c$ is a suitable constant, the sequence $\left(f_{j}\right)$ is uniformly bounded by $2 \int f_{i}(p) d p$ and converges in $C\left(\left[0, \tau_{0}\right] ; L^{1}\right)$ to a mild solution $f$ of $(3.10)$ (using (3.12) and induction), since

$$
\sup _{t \in\left[0, \tau_{0}\right]}\left|\left(f_{j+1}-f_{j}\right)(t, \cdot)\right|_{L^{1}} \leq k \tau_{0} \sup _{t \in\left[0, \tau_{0}\right]}\left|\left(f_{j}-f_{j-1}\right)(t, \cdot)\right|_{L^{1}}, \quad j \in \mathbb{N} .
$$

The nonnegative solution $f$ is unique in $C\left(\left[0, \tau_{0}\right] ; L^{1}\right)$ by the $L^{1}$-Lipschitz property $(3.12)$ of $C^{P}(\cdot, n)$. The time-interval $\left[0, \tau_{0}\right]$ can be so chosen that $m(t)=\Phi(n)(t) \geq \frac{1}{2} n_{*}$ uniformly for $n \in \mathcal{K}$ and $0 \leq t \leq \tau_{0}$.

The map $\Phi$ is continuous. Indeed, let $(n, \tilde{n}) \in \mathcal{K} \times \mathcal{K}$ and $m=\Phi(n)$ resp. $\tilde{m}=\Phi(\tilde{n})$. Then for $t \leq \tau_{0}$,

$$
\int|(f-\tilde{f})(t, p)| d p \leq k t\|n-\tilde{n}\|_{\infty}\left|+k \int_{0}^{t} \int\right|(f-\tilde{f})(s, p) \mid d p d s .
$$

Consequently, for $\tau$ small

$$
\sup _{t \in[0, \tau]} \int|(f-\tilde{f})(t, p)| d p \leq k \tau\|n-\tilde{n}\|_{\infty},
$$


and so

$$
\|m-\tilde{m}\|_{\infty} \leq k \tau\|n-\tilde{n}\|_{\infty} .
$$

The continuity of $\Phi$ on $\left[0, \tau_{0}\right]$ follows. Moreover, the map $\Phi$ is compact by Arzela-Ascoli. Indeed $\Phi(\mathcal{K})$ is bounded on $\left[0, \tau_{0}\right]$, since $\frac{1}{2} n_{*} \leq \Phi(n)(t) \leq M_{0}$ for $t \in\left[0, \tau_{0}\right], n \in \mathcal{K}$. Besides it is equicontinuous, since

$$
\begin{array}{r}
\left|\Phi(n)\left(t_{1}\right)-\Phi(n)\left(t_{2}\right)\right|=\left|\int f\left(t_{1}, p\right) d p-\int f\left(t_{2}, p\right) d p\right| \leq\left|t_{1}-t_{2}\right| \sup _{t \in\left[0, \tau_{0}\right]} \int|C(f, n)(t, p)| d p \\
\leq k\left(\sup _{t \in\left[0, \tau_{0}\right]} \int f(t, p) d p\left(P^{2}+\sup _{t \in\left[0, \tau_{0}\right]} \int f(t, p) d p\right)\right)\left|t_{1}-t_{2}\right| \\
\leq k\left(2 \int f_{i}(p) d p\left(P^{2}+2 \int f_{i}(p) d p\right)\right)\left|t_{1}-t_{2}\right|, \quad n \in K .
\end{array}
$$

Consequently, there is a pair of functions $\left(f^{P}, n_{c}^{P}\right) \in C\left(\left[0, \tau_{0}\right], L^{1}\right) \times C\left(\left[0, \tau_{0}\right]\right)$, such that $f^{P}$ is nonnegative and satisfies (3.10) in mild form with a truncation for $|p|>P$ in the linear part of the collision operator, and $n_{c}^{P}$ being a fixed point of $\Phi$, satisfies the corresponding equation (2.2) in mild form. Since $\int C^{P}\left(f^{P}, n_{c}^{P}\right) d p$ is continuous in $t$, the solution $n_{c}^{P}$ is continuously differentiable in $t$ and satisfies (2.2) in strong form. Also $f^{P}$ satisfies (2.1), (2.3) for $C^{P}$ in strong form since

Lemma 3.5 The family $\left(C^{P}\left(f^{P}, n^{P}\right)(t)\right), t \in\left[0, T_{0}\right]$, with values in $L^{1}$, is $t$-continuous in the $L^{1}$-norm, uniformly with respect to $P$ and $t$.

Proof of Lemma 3.5

Let us first discuss the time difference in the linear term from $C^{P}\left(f^{P}, n^{P}\right)(t+h)-C^{P}\left(f^{P}, n^{P}\right)(t)$, i.e.

$$
\begin{gathered}
\left.\int\left|\int d p_{1} d p_{2} d p_{3} \chi_{|p|<P}\right| \bar{A}\right|^{2} \delta\left(p_{1}-p_{2}-p_{3}\right) \delta\left(E_{1}-E_{2}-E_{3}\right)\left[\delta\left(p-p_{1}\right)-\delta\left(p-p_{2}\right)\right. \\
\left.-\delta\left(p-p_{3}\right)\right] f_{1}^{P}(t+h)-\int d p_{1} d p_{2} d p_{3} \chi_{|p|<P}|\bar{A}|^{2} \delta\left(p_{1}-p_{2}-p_{3}\right) \delta\left(E_{1}-E_{2}-E_{3}\right)\left[\delta\left(p-p_{1}\right)\right. \\
\left.-\delta\left(p-p_{2}\right)-\delta\left(p-p_{3}\right)\right] f_{1}^{P}(t) \mid d p .
\end{gathered}
$$

We move the difference through, one factor at a time. We recall that $\int\left(1+|p|^{2}\right) f^{P}(p, t) d p$ is uniformly in $P$ and $t$ bounded, and that $n^{P}(t)$ is $t$-continuous uniformly in $P$. When the factor $f_{1}^{P}(t+h)-f_{1}^{P}(t)$ appears, it is written as an integral $\int_{t}^{t+h} C^{P}\left(f^{P}, n^{P}\right)(s) d s$, using the mild form of the equation. Here $C^{P}\left(f^{P}, n^{P}\right)$ can be estimated similarly to the proof of Lemma 3.1. Consider again the linear term

$$
\begin{gathered}
\int_{t}^{t+h} d s \int d p \int d p_{1} d p_{2} d p_{3} \chi_{|p|<P}|\bar{A}|^{2} \delta\left(p_{1}-p_{2}-p_{3}\right) \delta\left(E_{1}-E_{2}-E_{3}\right) \delta\left(p-p_{1}\right) \\
\int \chi_{\left|p_{1}\right|<P}|\bar{A}|^{2} \delta\left(p_{1}^{\prime}-p_{2}^{\prime}-p_{3}^{\prime}\right) \delta\left(E_{1}^{\prime}-E_{2}^{\prime}-E_{3}^{\prime}\right) \delta\left(p_{1}-p_{1}^{\prime}\right) f\left(p_{1}^{\prime}, s\right) d p_{1}^{\prime} d p_{2}^{\prime} d p_{3}^{\prime} .
\end{gathered}
$$

Use the computation of $X_{3}$ in the proof of Lemma 3.1 to estimate the outer integral with respect to $d p d p_{1} d p_{2} d p_{3}$. This gives a bound

$$
\begin{aligned}
k \int_{0}^{\infty} & d r_{1} r_{1} \int_{0}^{r_{1}} r_{2} d r_{2} \int|\bar{A}|^{2} \delta\left(p_{1}^{\prime}-p_{2}^{\prime}-p_{3}^{\prime}\right) \delta\left(E_{1}^{\prime}-E_{2}^{\prime}-E_{3}^{\prime}\right) \delta\left(p_{1}-p_{1}^{\prime}\right) f\left(p_{1}^{\prime}, s\right) d p_{1}^{\prime} d p_{2}^{\prime} d p_{3}^{\prime} \\
& =k \int_{0}^{\infty} d r_{1} r_{1}^{3} \int|\bar{A}|^{2} \delta\left(p_{1}^{\prime}-p_{2}^{\prime}-p_{3}^{\prime}\right) \delta\left(E_{1}^{\prime}-E_{2}^{\prime}-E_{3}^{\prime}\right) \delta\left(p_{1}-p_{1}^{\prime}\right) f\left(p_{1}^{\prime}, s\right) d p_{1}^{\prime} d p_{2}^{\prime} d p_{3}^{\prime} \\
& =k \int|\bar{A}|^{2} \delta\left(p_{1}^{\prime}-p_{2}^{\prime}-p_{3}^{\prime}\right) \delta\left(E_{1}^{\prime}-E_{2}^{\prime}-E_{3}^{\prime}\right) f\left(p_{1}^{\prime}, s\right) d p_{1}^{\prime} d p_{2}^{\prime} d p_{3}^{\prime} \int\left|p_{1}\right| \delta\left(p_{1}-p_{1}^{\prime}\right) d p_{1}
\end{aligned}
$$


Again using the $X_{3}$-computation for the remaining integral gives the bound

$$
k \int_{0}^{\infty} d r_{1}^{\prime} r_{1}^{\prime 2} f\left(r_{1}^{\prime}, s\right) \int_{0}^{r_{1}^{\prime}} d r_{2}^{\prime} r_{2}^{\prime} \leq k \int d p_{1}^{\prime}\left|p_{1}^{\prime}\right|^{2} f\left(p_{1}^{\prime}, s\right) .
$$

And so the final integration $\int_{t}^{t+h} d s$ gives the bound $h k \sup _{[t, t+h]} \int p^{2} f(p, s) d p$. It follows that (3.15), uniformly in $P$ and $t, L^{1}$-converges to zero when $h \rightarrow 0$. That holds in a similar way for the other terms in (3.14), and for the non-linear terms of $C^{P}\left(f^{P}, n^{P}\right)$.

The conservation of total mass follows from the fixed point property. The boundedness of the energy of $f^{P}$ follows from the proof of Lemma 3.1. Using (3.4) the integrals $\int\left(1+p^{2}\right) f^{P} d p$ are also uniformly in $P$ bounded. Moreover, the estimate of Lemma 3.4 holds uniformly in $P$ for $f^{P}$. Observing that $n_{*}$ is so chosen that for any $P, n_{c}^{P} \geq n_{*}$ on any subinterval $\left[0, T_{0}^{\prime}\right]$ of $\left[0, T_{0}\right]$ where $n_{c}^{P}$ exists, the result can for each $P$ be extended by iteration to the whole interval of time $\left[0, T_{0}\right]$.

The $f^{P}$ 's are also the limits in $C\left(\left[0, T_{0}\right] ; L_{1+p^{2}}^{1}\right)$ of increasing sequences $\left(\tilde{f}_{j}^{P}\right)$. It will be used in the study of $\lim _{P \rightarrow \infty} f^{P}$ below, that such $\tilde{f}_{j}^{P}$ 's share with the $f^{P}$ 's any uniform bound for $(2+\gamma)$ moments. The increasing sequences of approximations are defined by $\tilde{f}_{0}^{P}=0$ and

$$
\begin{array}{r}
\frac{\partial \tilde{f}_{j+1}^{P}}{\partial t}+\tilde{f}_{j+1}^{P}\left(n_{c}^{P} \int|\bar{A}|^{2} \chi_{|p|<P} \delta\left(p-p_{2}-p_{3}\right) \delta\left(E_{p}-E_{2}-E_{3}\right) d p_{2} d p_{3}+\tilde{C}_{l}\left(f^{P}, n_{c}^{P}\right)\right)=\tilde{C}_{g}\left(\tilde{f}_{j}^{P}, n_{c}^{P}\right) \\
+2 n_{c}^{P} \int|\bar{A}|^{2} \chi_{|p|<P} \delta\left(p_{1}-p_{2}-p\right) \delta\left(E_{1}-E_{2}-E_{p}\right) f_{1}^{P} d p_{1} d p_{2},(3.16 \\
\tilde{f}_{j+1}^{P}(0, p)=f_{i}(p) \chi_{|p|<P}
\end{array}
$$

Here $\tilde{C}_{l}$ is defined by

$$
\begin{aligned}
\tilde{C}_{l}(f, n)= & 2 n\left[\int|\bar{A}|^{2} \delta\left(p-p_{2}-p_{3}\right) \delta\left(E_{p}-E_{2}-E_{3}\right) f_{2} d p_{2} d p_{3}\right. \\
& \left.+\int|\bar{A}|^{2} \delta\left(p_{1}-p_{2}-p\right) \delta\left(E_{1}-E_{2}-E_{p}\right) f_{2} d p_{1} d p_{2}\right]
\end{aligned}
$$

and

$$
\begin{aligned}
\tilde{C}_{g}(f, n)= & n\left[\int|\bar{A}|^{2} \delta\left(p-p_{2}-p_{3}\right) \delta\left(E_{p}-E_{2}-E_{3}\right) f_{2} f_{3} d p_{2} d p_{3}\right. \\
& +2 \int|\bar{A}|^{2} \delta\left(p_{1}-p_{2}-p\right) \delta\left(E_{1}-E_{2}-E_{p}\right) f_{1} f_{2} d p_{1} d p_{2} \\
& \left.+2 f \int|\bar{A}|^{2} \delta\left(p_{1}-p_{2}-p\right) \delta\left(E_{1}-E_{2}-E_{p}\right) f_{1} d p_{1} d p_{2}\right]
\end{aligned}
$$

Let us prove by induction that $\left(\tilde{f}_{j}^{P}\right), j \in \mathbb{N}$, is an increasing sequence of nonnegative functions bounded by $f^{P}$. First, $0 \leq \tilde{f}_{0}^{P} \leq f^{P}$ and $\tilde{f}_{0}^{P} \leq \tilde{f}_{1}^{P}$, by definition of $\tilde{f}_{0}^{P}$ and the nonnegativity of $f_{\tilde{\sim}}^{P}$ and $\tilde{f}_{1}^{P}$. Then, assuming that $\tilde{f}_{j-1}^{P} \leq \tilde{f}_{j}^{P}$ and $\tilde{f}_{j-1}^{P} \leq f^{P}$, the function $\tilde{f}_{j+1}^{P}-\tilde{f}_{j}^{P}$ (resp. $\left.f^{P}-\tilde{f}_{j}^{P}\right)$ satisfies

$$
\begin{array}{r}
\frac{\partial\left(\tilde{f}_{j+1}^{P}-\tilde{f}_{j}^{P}\right)}{\partial t}+\left(\tilde{f}_{j+1}^{P}-\tilde{f}_{j}^{P}\right)\left(n_{c}^{P} \int|\bar{A}|^{2} \chi_{|p|<P} \delta\left(p-p_{2}-p_{3}\right) \delta\left(E_{p}-E_{2}-E_{3}\right) d p_{2} d p_{3}+\tilde{C}_{l}\left(f^{P}, n_{c}^{P}\right)\right) \\
=\tilde{C}_{g}\left(\tilde{f}_{j}^{P}, n_{c}^{P}\right)-\tilde{C}_{g}\left(\tilde{f}_{j-1}^{P}, n_{c}^{P}\right), \\
\left(\tilde{f}_{j+1}^{P}-\tilde{f}_{j}^{P}\right)(0, p)=0,
\end{array}
$$


(resp.

$$
\begin{array}{r}
\frac{\partial\left(f^{P}-\tilde{f}_{j}^{P}\right)}{\partial t}+\left(f^{P}-\tilde{f}_{j}^{P}\right)\left(n_{c}^{P} \int|\bar{A}|^{2} \chi_{|p|<P} \delta\left(p-p_{2}-p_{3}\right) \delta\left(E_{p}-E_{2}-E_{3}\right) d p_{2} d p_{3}+\tilde{C}_{l}\left(f^{P}, n_{c}^{P}\right)\right) \\
=\tilde{C}_{g}\left(f^{P}, n_{c}^{P}\right)-\tilde{C}_{g}\left(\tilde{f}_{j-1}^{P}, n_{c}^{P}\right), \\
\left.\left(f^{P}-\tilde{f}_{j}^{P}\right)(0, p)=0 .\right)
\end{array}
$$

Writing $\left(\tilde{f}_{j+1}^{P}-\tilde{f}_{j}^{P}\right)(t, p)$ (resp. $\left.\left(f^{P}-\tilde{f}_{j}^{P}\right)(t, p)\right)$ in exponential form, and deducing from the induction assumption that $\tilde{C}_{g}\left(\tilde{f}_{j}^{P}, n_{c}^{P}\right)-\tilde{C}_{g}\left(\tilde{f}_{j-1}^{P}, n_{c}^{P}\right)\left(\operatorname{resp} . \tilde{C}_{g}\left(\tilde{f}_{j}^{P}, n_{c}^{P}\right)-\tilde{C}_{g}\left(\tilde{f}_{j-1}^{P}, n_{c}^{P}\right)\right)$ is nonnegative, leads to the nonnegativity of $\left(\tilde{f}_{j+1}^{P}-\tilde{f}_{j}^{P}\right)(t, p)$ (resp. $\left.\left(\tilde{f}^{P}-\tilde{f}_{j}^{P}\right)(t, p)\right)$. Its limit $\tilde{f}^{P}$ satisfies the same equation as $f^{P}$. The collision operator for the equation satisfied by $f^{P}-\tilde{f}^{P}$ is Lipschitz continuous similarly to (3.12), hence $f^{P}=\tilde{f}^{P}$.

It remains to prove that a subsequence of $\left(f^{P}, n_{c}^{P}\right)$ converges in $C\left(\left[0, T_{0}\right], L_{1+p^{2}}^{1}\right) \times C\left(\left[0, T_{0}\right]\right)$, and that its limit solves the system (2.1-3). Using Arzela-Ascoli as above, the sequence $\left(n^{P}\right)$ is compact in $C\left(\left[0, T_{0}\right]\right)$.

Lemma 3.6 Given $t \in\left[0, T_{0}\right]$, the family $\left(g^{P}(t)\right):=\left(\int|\bar{A}|^{2} \chi_{|p|<P} \delta\left(p_{1}-p_{2}-p\right) \delta\left(E_{1}-E_{2}-\right.\right.$ $\left.\left.E_{p}\right) f_{1}^{P}(t) d p_{1} d p_{2}\right)$ is compact in $L^{1}$. This also holds for the family $\left(\tilde{C}_{l}\left(f^{P}, n_{c}^{P}\right)(t)\right)$.

Proof of Lemma 3.6.

By definition,

$$
\begin{array}{r}
g^{P}(p)=\chi_{|p|<P} \int|\bar{A}|^{2} f_{1}^{P} \delta\left(p_{1}=p_{2}+p\right) \delta\left(E_{1}=E_{2}+E_{p}\right) d p_{1} d p_{2} \\
=\chi_{|p|<P} \int|\bar{A}|^{2} r_{1}^{2} f_{1}^{P}\left(r_{1}\right) \int_{-1}^{1} \delta(F(s)=0) d s d r_{1}
\end{array}
$$

where

$$
F(s):=\sqrt{\left(2|p| r_{1} s-r_{1}^{2}-p^{2}\right)^{2}-n\left(2|p| r_{1} s-r_{1}^{2}-p^{2}\right)}+E_{p}-E_{1} .
$$

Then,

$$
\begin{array}{r}
F(s)=0 \text { iif }\left(2|p| r_{1} s-r_{1}^{2}-p^{2}\right)^{2}-n\left(2|p| r_{1} s-r_{1}^{2}-p^{2}\right)-\left(E_{p}-E_{1}\right)^{2}=0 \text { and } r_{1} \geq|p| \\
\quad \text { iif } 2|p| r_{1} s-r_{1}^{2}-p^{2}=\frac{n-\sqrt{n^{2}+4\left(E_{p}-E_{1}\right)^{2}}}{2}=: 2|p| r_{1} \tilde{s}-r_{1}^{2}-p^{2} \text { and } r_{1} \geq|p| .
\end{array}
$$

Moreover,

$$
F^{\prime}(s)=\frac{|p| r_{1}\left(2\left(2|p| r_{1} s-r_{1}^{2}-p^{2}\right)-n\right)}{F(s)-\left(E_{p}-E_{1}\right)}
$$

so that

$$
\left|F^{\prime}(\tilde{s})\right|=\frac{|p| r_{1} \sqrt{n^{2}+4\left(E_{p}-E_{1}\right)^{2}}}{\left|E_{p}-E_{1}\right|} .
$$

And so, up to an angular factor,

$$
\begin{array}{r}
g^{P}(p)=\frac{\chi_{|p|<P}}{|p|} \int_{|p|}^{+\infty}|\bar{A}|^{2} r_{1} f_{1}^{P} \frac{\left|E_{p}-E_{1}\right|}{\sqrt{n^{2}+4\left(E_{p}-E_{1}\right)^{2}}} \chi_{|\tilde{s}| \leq 1} d r_{1} \\
=\frac{\chi_{|p|<P}}{|p|} \int_{|p|}^{+\infty}|\bar{A}|^{2} r_{1} f_{1}^{P} \frac{\left|E_{p}-E_{1}\right|}{\sqrt{n^{2}+4\left(E_{p}-E_{1}\right)^{2}}} \chi_{\left|2 r_{1}^{2}+2 p^{2}+n-\sqrt{n^{2}+4\left(E_{p}-E_{1}\right)^{2}}\right| \leq 4|p| r_{1}} d r_{1} .
\end{array}
$$


Moreover,

$$
\left|2 r_{1}^{2}+2 p^{2}+n-\sqrt{n^{2}+4\left(E_{p}-E_{1}\right)^{2}}\right| \leq 4|p| r_{1}
$$

if and only if

$$
\sqrt{n^{2}+4\left(E_{p}-E_{1}\right)^{2}} \leq 2 r_{1}^{2}+4 r r_{1}+2 r^{2}+n
$$

and

$$
2 r_{1}^{2}-4 r r_{1}+2 r^{2}+n \leq \sqrt{n^{2}+4\left(E_{p}-E_{1}\right)^{2}} .
$$

Straightforward computations show that the first inequality is satisfied for any $\left(n, p, p_{1}\right)$. The second inequality is equivalent to

$$
r_{1}^{4}-3 r r_{1}^{3}+\left(4 r^{2}+\frac{3}{4} n\right) r_{1}^{2}-3 r\left(r^{2}+\frac{n}{2}\right) r_{1}+r^{4}+\frac{3}{4} n r^{2} \geq 0,
$$

which is also true for any $\left(n, p_{1}, p\right)$, since its left hand side is equal to

$$
\left(r_{1}-r\right)^{4}+\left(r_{1}-r\right)^{2}\left(r_{1} r+\frac{3}{4} n\right) .
$$

And so,

$$
g^{P}(p)=\frac{\chi_{|p|<P}}{|p|} \int_{|p|}^{+\infty}|\bar{A}|^{2}\left(p_{1}, p_{1}-p, p\right) r_{1} f_{1}^{P} \frac{\left|E_{p}-E_{1}\right|}{\sqrt{n^{2}+4\left(E_{p}-E_{1}\right)^{2}}} d r_{1} .
$$

We next prove the $L^{1}$-compactness of $\left(g^{P}\right)(t)$. First,

$$
\lim _{K \rightarrow+\infty} \int_{|p|>K} g^{P}(p) d p=0 .
$$

Indeed,

$$
\begin{gathered}
\int_{|p|>K} g^{P}(p) d p \leq k \int_{K}^{+\infty} r_{1} f^{P}\left(r_{1}\right) \int_{K}^{r_{1}} r_{3} d r_{3} d r_{1} \\
\leq k \int_{K}^{+\infty} r_{1}^{3} f^{P}\left(r_{1}\right) d r_{1} \leq \frac{k}{K} \int p^{2} f^{P}(p) d p
\end{gathered}
$$

which uniformly in $P$ and $t$ tends to zero when $K \rightarrow+\infty$.

Let us prove that for fixed $K>0$,

$$
\lim _{h \rightarrow 0} \int\left|g^{P}(p+h) \chi_{|p+h|<K}-g^{P}(p) \chi_{|p|<K}\right| d p=0,
$$

uniformly with respect to $P$ and $t$. It holds that

$$
\begin{array}{r}
\int_{|p|<K+1}\left|g^{P}(p+h)-g^{P}(p)\right| d p \leq M_{0} \int_{|p|<K+1}\left|\frac{\chi|p+h|<P}{|p+h|}-\frac{\chi|p|<P}{|p|}\right| \frac{d p}{|p|} \\
+\int f_{1}^{P} \int_{|p|<K+1}\left|\bar{A}^{2}\left(p_{1}, p_{1}-p-h, p+h\right)\right| \frac{\left|E_{p+h}-E_{1}\right|}{\sqrt{n^{2}+4\left(E_{p+h}-E_{1}\right)^{2}}} \\
-\left|\bar{A}^{2}\left(p_{1}, p_{1}-p, p\right)\right| \frac{\left|E_{p}-E_{1}\right|}{\sqrt{n^{2}+4\left(E_{p}-E_{1}\right)^{2}}} \mid \frac{d p}{|p|} d p_{1}+O(h) .
\end{array}
$$


The inner $p$-integral in the last term of the previous inequality tends to zero when $h$ tends to zero, uniformly with respect to $p_{1}$, and so (3.17) follows.

For the family $\tilde{C}_{l}\left(f^{P}, n_{c}^{P}\right)$ it is enough to consider a sequence $\left(P_{j}\right)$ tending to infinity, for which $\left(n_{c}^{P_{j}}\right)$ is uniformly in $t$ convergent. From there the proof is similar to the previous case, since

$$
\begin{aligned}
\tilde{C}_{l}\left(f^{P}, n_{c}^{P}\right)(p)= & n^{P}\left(\frac{2}{|p|} \int_{0}^{|p|}|\bar{A}|^{2} r_{2} f_{2}^{P} \frac{\left|E_{p}-E_{2}\right|}{\sqrt{n^{2}+4\left(E_{p}-E_{2}\right)^{2}}} d r_{2}\right. \\
& \left.+\frac{2}{|p|} \int_{0}^{+\infty}|\bar{A}|^{2} r_{2} f_{2}^{P} \frac{E_{2}+E_{p}}{\sqrt{n^{2}+4\left(E_{2}+E_{p}\right)^{2}}} d r_{2}\right) .
\end{aligned}
$$

We can now take a subsequence $\left(g^{P_{l}}, \tilde{C}_{l}\left(f^{P_{l}}, n_{c}^{P_{l}}\right), n_{c}^{P_{l}}\right)$ with $P_{l}$ tending to infinity, converging for rational $t$ to a limit $\left(g, h, n_{c}\right)$, and will for such $t$ prove that $\left(f^{P_{l}}\right)$ is a Cauchy sequence in $L^{1}$. The Cauchy property for irrational $t$ then follows using Lemma 3.5.

To prove that $\left(f^{P_{l}}\right)$ is a Cauchy sequence in $L^{1}$, split $f^{P_{l^{\prime}}}-f^{P_{l} \text { " into }}$

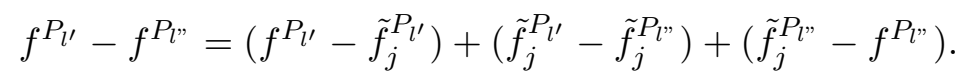

By the estimate of Lemma 3.4 it is enough to prove the convergence on compact $p$-sets. On any such set the factor $\int|\bar{A}|^{2} \chi_{|p|<P} \delta\left(p-p_{2}-p_{3}\right) \delta\left(E_{p}-E_{2}-E_{3}\right) d p_{2} d p_{3}$ in the left hand side of (3.16) does not depend on $P$ for $P$ large enough.

It follows from the equations (3.10) and (3.16) for $f^{P_{l^{\prime}}}-\tilde{f}_{j+1}^{P_{l^{\prime}}}$, and from the cancelation of the inhomogeneous term in the right hand side of the equation, that

$$
\lim _{j \rightarrow+\infty}\left(f^{P_{l^{\prime}}-\tilde{f}_{j}^{P^{\prime}}}\right)=0,
$$

uniformly with respect to $l^{\prime}$ and $t$. It remains to prove that for $J$ given,

$$
\lim _{l^{\prime} \rightarrow+\infty, l^{\prime \prime} \rightarrow+\infty}\left(\tilde{f}_{J}^{P_{l^{\prime}}}-\tilde{f}_{J}^{P_{l}^{\prime \prime}}\right)=0
$$

in $L^{1}$-sense. Consider first the case $J=1$. In (3.16) for $\tilde{f}_{1}^{P_{l^{\prime}}}$, the term

$$
\tilde{f}_{1}^{P_{l^{\prime}}} n_{c}^{P_{l^{\prime}}} \int|\bar{A}|^{2} \chi_{|p|<P} \delta\left(p-p_{2}-p_{3}\right) \delta\left(E_{p}-E_{2}-E_{3}\right) d p_{2} d p_{3}
$$

to the left can be written for $n_{c}$, plus a term in $n_{c}-n_{c} P_{l^{\prime}}$, which tends to zero in $L^{1}$, when $l^{\prime} \rightarrow \infty$. The $L^{1}$ limit of the right hand side is $n_{c} g$. So using the $L^{1}$-convergence of the $\tilde{C}_{l^{\prime}}$-term, $(3.16)$ gives that $\tilde{f}_{1}^{P_{l^{\prime}}}$ converges in $L^{1}$ to some $\tilde{f}_{1}$. It follows that $\tilde{C}_{g}\left(\tilde{f}_{1}^{P_{l}}, n_{c}^{P_{1}}\right)$ converges in $L^{1}$ to $\tilde{C}_{g}\left(\tilde{f}_{1}, n_{c}\right)$. We can now repeat the convergence argument for $\tilde{f}_{2}^{P_{l^{\prime}}}$. By finite induction for $j=1, \ldots, J$, the desired convergence holds. Also $f=\lim f^{P_{l}} \in L^{1}$, and

$$
\begin{array}{r}
\frac{\partial f}{\partial t}+f\left(n_{c} \int|\bar{A}|^{2} \chi \delta\left(p-p_{2}-p_{3}\right) \delta\left(E_{p}-E_{2}-E_{3}\right) d p_{2} d p_{3}+h\right)=\tilde{C}_{g}\left(f, n_{c}\right)+2 n_{c} g \\
f(0, p)=f_{i}(p) .
\end{array}
$$

But the $L^{1}$-convergence of $\left(f^{P_{l}}\right)$ and Lemma 3.4 imply that

$$
\begin{array}{r}
g=\lim _{l \rightarrow \infty} g^{P_{l}}=\lim _{l \rightarrow \infty} \int|\bar{A}|^{2} \chi \chi_{|p|<P} \delta\left(p_{1}-p_{2}-p\right) \delta\left(E_{1}-E_{2}-E_{p}\right) f_{1}^{P} d p_{1} d p_{2} \\
=\int|\bar{A}|^{2} \chi \delta\left(p_{1}-p_{2}-p\right) \delta\left(E_{1}-E_{2}-E_{p}\right) f_{1} d p_{1} d p_{2} \\
h=\lim _{l \rightarrow \infty} \tilde{C}_{l}\left(f^{P_{l}}, n_{c}^{P_{l}}\right)=\tilde{C}_{l}\left(f, n_{c}\right) .
\end{array}
$$


And so $\left(f, n_{c}\right)$ satisfies (2.1-3) on $\left[0, T_{0}\right]$ in mild form with the $|p|^{2+\gamma}$-moment of $f$ bounded in $L^{1}$. This can be continued up to any time $T$, since by $(3.5) \inf _{t \in[0, T[} n_{c}(t)>0$ for any time interval $[0, T[$ where $\left(f, n_{c}\right)$ exists. The $C^{1}$-properties of $f, n_{c}$ with respect to time, follow as above for $f^{P}, n_{c}^{P}$.

\section{The special cases $|p|<<p_{0}$ and $|p|>>p_{0}$.}

In the case $|p|<<p_{0}$ the collision operator becomes

$$
\begin{array}{r}
C\left(f, n_{c}\right)=k n_{c} \int_{\left|p_{i}\right| \leq \lambda} \frac{E\left(p_{1}\right) E\left(p_{2}\right) E\left(p_{3}\right)}{\left(g n_{c}\right)^{3}} \delta\left(p_{1}-p_{2}-p_{3}\right) \delta\left(E_{1}-E_{2}-E_{3}\right)\left[\delta\left(p-p_{1}\right)\right. \\
\left.-\delta\left(p-p_{2}\right)-\delta\left(p-p_{3}\right)\right]\left(\left(1+f_{1}\right) f_{2} f_{3}-f_{1}\left(1+f_{2}\right)\left(1+f_{3}\right)\right) d p_{1} d p_{2} d p_{3} \\
=k n_{c}^{-\frac{1}{2}} \int_{\left|p_{i}\right| \leq \lambda}\left|p_{1}\right|\left(1+\frac{p_{1}^{2}}{4 p_{0}^{2}}\right)\left|p_{2}\right|\left(1+\frac{p_{2}^{2}}{4 p_{0}^{2}}\right)\left|p_{3}\right|\left(1+\frac{p_{3}^{2}}{4 p_{0}^{2}}\right) \delta\left(p_{1}-p_{2}-p_{3}\right) \delta\left(E_{1}-E_{2}-E_{3}\right)\left[\delta\left(p-p_{1}\right)\right. \\
\left.-\delta\left(p-p_{2}\right)-\delta\left(p-p_{3}\right)\right]\left(f_{2} f_{3}-f_{1}\left(1+f_{2}+f_{3}\right)\right) d p_{1} d p_{2} d p_{3} \\
=k n_{c}^{-\frac{1}{2}} \int_{\left|p_{i}\right| \leq \lambda} \tilde{E}_{1} \tilde{E}_{2} \tilde{E}_{3} \delta\left(p_{1}-p_{2}-p_{3}\right) \delta\left(c\left(\tilde{E}_{1}-\tilde{E}_{2}-\tilde{E}_{3}\right)\right)\left[\delta\left(p-p_{1}\right)\right. \\
\left.-\delta\left(p-p_{2}\right)-\delta\left(p-p_{3}\right)\right]\left(f_{2} f_{3}-f_{1}\left(1+f_{2}+f_{3}\right)\right) d p_{1} d p_{2} d p_{3},
\end{array}
$$

with $\tilde{E}(|p|)=|p|\left(1+\frac{p^{2}}{4 p_{0}^{2}}\right)$.

Global conservation of total mass and of momentum are obtained as in the general case. And so energy is globally bounded, since the $p$-domain is bounded.

\section{Lemma 4.1}

$$
\left|\int C\left(f, n_{c}\right)(p) d p\right| \leq k n_{c}\left(\int f(p) d p\right)\left(1+\int f(p) d p\right)
$$

Proof of Lemma 4.1

$$
\begin{array}{r}
\left|\int C\left(f, n_{c}\right)(p) d p\right| \leq k n_{c}{ }^{-2} \int_{\left|p_{i}\right|<\lambda} E\left(p_{1}\right) E\left(p_{2}\right) E\left(p_{3}\right) \\
\delta\left(p_{1}-p_{2}-p_{3}\right) \delta\left(E\left(p_{1}\right)-E\left(p_{2}\right)-E\left(p_{3}\right)\right)\left(f_{2} f_{3}+f_{1}\left(1+f_{2}+f_{3}\right)\right) d p_{1} d p_{2} d p_{3} \\
=k n_{c}^{-1} \int_{\left|p_{i}\right| \leq \lambda} \tilde{E}_{1} \tilde{E}_{2} \tilde{E}_{3} \delta\left(p_{1}-p_{2}-p_{3}\right) \delta\left(\tilde{E}_{1}-\tilde{E}_{2}-\tilde{E}_{3}\right)\left(f_{2} f_{3}+f_{1}\left(1+f_{2}+f_{3}\right)\right) d p_{1} d p_{2} d p_{3} .
\end{array}
$$

For controlling the $f_{2} f_{3}$ term of (4.1), use spherical coordinates for $p_{2}$ and $p_{3}$ with the axis for $p_{3}$ directed by $p_{2}$ and denote by $\varphi_{3}$ the azimuthal angle related to $p_{3}$. Then

$$
\begin{aligned}
n_{c}^{-1} \int \chi \tilde{E}\left(p_{1}\right) \tilde{E}\left(p_{2}\right) \tilde{E}\left(p_{3}\right) \delta\left(p_{1}-\right. & \left.\left.p_{2}-p_{3}\right)\right) \delta\left(\tilde{E}\left(p_{1}\right)-\tilde{E}\left(p_{2}\right)-\tilde{E}\left(p_{3}\right)\right) f\left(p_{2}\right) f\left(p_{3}\right) d p_{1} d p_{2} d p_{3} \\
& =k n_{c}^{-1} \int_{0}^{\lambda} r_{2}^{2} \tilde{E}_{2} f\left(p_{2}\right) \int_{0}^{r_{2}} r_{3}^{2} \tilde{E}_{3}\left(\tilde{E}_{2}+\tilde{E}_{3}\right) f\left(p_{3}\right) X d r_{2} d r_{3}
\end{aligned}
$$


where

$$
X=\int_{-1}^{1} \chi \delta\left(\tilde{E}\left(\sqrt{r_{2}^{2}+r_{3}^{2}+2 r_{2} r_{3} s}\right)-\tilde{E}_{2}-\tilde{E}_{3}\right) d s \leq \frac{k n_{c}}{r_{2} r_{3}},
$$

since

$$
\begin{array}{r}
\delta\left(\tilde{E}\left(\sqrt{r_{2}^{2}+r_{3}^{2}+2 r_{2} r_{3} s}\right)-\tilde{E}_{2}-\tilde{E}_{3}\right)=\frac{\sqrt{r_{2}^{2}+r_{3}^{2}+2 r_{2} r_{3} s}}{r_{2} r_{3} \tilde{E}^{\prime}\left(\sqrt{r_{2}^{2}+r_{3}^{2}+2 r_{2} r_{3} s}\right)} \delta(s=\tilde{s}) \\
=\frac{4 p_{0}^{2} \sqrt{r_{2}^{2}+r_{3}^{2}+2 r_{2} r_{3} s}}{r_{2} r_{3}\left(4 p_{0}^{2}+3\left(r_{2}^{2}+r_{3}^{2}+2 r_{2} r_{3} s\right)\right)} \delta(s=\tilde{s}),
\end{array}
$$

and

$$
\frac{4 p_{0}^{2} r}{4 p_{0}^{2}+3 r^{2}} \leq k r \leq k n_{c}, \quad 0 \leq r \leq \lambda .
$$

And so,

$$
\begin{array}{r}
\left.k n_{c}^{-1} \int \chi \tilde{E}\left(p_{1}\right) \tilde{E}\left(p_{2}\right) \tilde{E}\left(p_{3}\right) \delta\left(p_{1}-p_{2}-p_{3}\right)\right) \delta\left(\tilde{E}\left(p_{1}\right)-\tilde{E}\left(p_{2}\right)-\tilde{E}\left(p_{3}\right)\right) f\left(p_{2}\right) f\left(p_{3}\right) d p_{1} d p_{2} d p_{3} \\
\leq k \int_{0}^{\lambda} r_{2} \tilde{E}_{2}^{2} f_{2} d r_{2} \int_{0}^{\lambda} r_{3} \tilde{E}_{3} f_{3} d r_{3} \leq k n_{c}\left(\int f(p) d p\right)^{2} .
\end{array}
$$

In the same way the other terms of (4.1) can be bounded by $k n_{c} \int f(p) d p$ or $k n_{c}\left(\int f(p) d p\right)^{2}$.

Lemma 4.2 Given $0<n_{*}<M_{0}$, there is a constant $k$ such that for any $n \in\left[n_{*}, M_{0}\right]$ and isotropic functions $(f, g) \in L_{+}^{1}\left(\mathbb{R}^{3}\right) \times L_{+}^{1}\left(\mathbb{R}^{3}\right)$ with $L^{1}$ norm bounded by $M_{0}$,

$$
\int|(C(f, n)-C(g, n))(p)| d p \leq k \int|(f-g)(p)| d p
$$

with $k$ independent of $n, f, g$.

Proof of Lemma 4.2

Denote by $\mu(f)=\left(f_{2} f_{3}-f_{1}\left(1+f_{2}+f_{3}\right)\right)$. Then by computations similar to those used in the proof of Lemma 4.1,

$$
\begin{array}{rr}
\leq \frac{k}{n} \int \chi \tilde{E}_{1} \tilde{E}_{2} \tilde{E}_{3} \delta\left(p_{1}-p_{2}-p_{3}\right) \delta\left(\tilde{E}_{1}-\tilde{E}_{2}-\tilde{E}_{3}\right)|\mu(f)-\mu(g)| d p_{1} d p_{2} d p_{3} \\
\leq k n\left(1+\int_{|p| \leq \lambda} f d p+\int_{|p| \leq \lambda} g d p\right) \int_{|p| \leq \lambda}|f(p)-g(p)| d p .
\end{array}
$$

Lemma 4.3 Given $T>0$, consider the problem (2.1-3) on the interval $[0, T]$ with $0<n_{c i}<M_{0}$ and $n_{c i}+\int f_{i}(|p|) d p=M_{0}$. Then there is $n_{*}>0$ such that $n(t) \geq n_{*}$ on $[0, T]$ for any solution $\left(f, n_{c}\right)$. 


\section{Proof of Lemma 4.3}

We know from Lemma 4.1 that

$$
\left|\int C\left(f, n_{c}\right)(t, p) d p\right| \leq k n_{c}(t)\left(\int f(t, p) d p\right)\left(1+\int f(t, p) d p\right) \leq k M_{0}\left(1+M_{0}\right) n_{c}(t) .
$$

It follows that

$$
\frac{d n_{c}}{d t}=-n_{c} \cdot \frac{1}{n_{c}} \int C\left(f, n_{c}\right) d p
$$

with $\frac{1}{n_{c}} \int C\left(f, n_{c}\right) d p$ bounded on $[0, T]$. And so $n_{c}$ is at most exponentially decreasing with respect to time. The lemma follows.

After these preparations the proof of Theorem 2.2 becomes a simplified version of the proof of Theorem 2.1.

For the intermediate temperature case of Theorem 2.3, we take

$$
\begin{array}{r}
|\bar{A}|^{2}=1 \text { for }|p| \geq \alpha,\left|p_{1}\right| \geq \alpha,\left|p_{2}\right| \geq \alpha,\left|p_{3}\right| \geq \alpha, \\
|\bar{A}|^{2}=0 \quad \text { otherwise }
\end{array}
$$

with the corresponding collision operator

$$
\begin{gathered}
C\left(f, n_{c}\right)=n_{c} \int_{\mathbb{R}^{3} \times \mathbb{R}^{3} \times \mathbb{R}^{3}}|\bar{A}|^{2} \delta\left(p_{1}-p_{2}-p_{3}\right) \delta\left(E_{1}-E_{2}-E_{3}\right)\left[\delta\left(p-p_{1}\right)\right. \\
\left.-\delta\left(p-p_{2}\right)-\delta\left(p-p_{3}\right)\right]\left(\left(1+f_{1}\right) f_{2} f_{3}-f_{1}\left(1+f_{2}\right)\left(1+f_{3}\right)\right) d p_{1} d p_{2} d p_{3}
\end{gathered}
$$

Global conservation of total mass and of momentum are obtained as in the general case. Moreover, multiplying (2.1) by $p^{2}$ and integrating, leads to

$$
\begin{array}{r}
\frac{d}{d t} \int p^{2} f(t, p) d p=n_{c} \int \chi \delta\left(p_{1}-p_{2}-p_{3}\right) \delta\left(p_{1}^{2}=p_{2}^{2}+p_{3}^{2}+n_{c}\right)\left(\left|p_{1}\right|^{2}-\left|p_{2}\right|^{2}-\left|p_{3}\right|^{2}\right) \\
\left(f_{2} f_{3}-f_{1}\left(1+f_{2}+f_{3}\right)\right) d p_{1} d p_{2} d p_{3}=-2 m g n_{c} \int C\left(f, n_{c}\right)(t, p) d p=2 m g n_{c} n_{c}^{\prime} .
\end{array}
$$

Hence,

$$
\int p^{2} f(t, p) d p=\int p^{2} f_{i}(p) d p+m g n_{c}^{2}(t)-m g n_{c i}^{2} .
$$

This implies that the integral

$$
\int p^{2} f(t, p) d p+m g n_{c}(t) \int f(t, p) d p+m g M_{0}\left(2 \int f(t, p) d p+n_{c}(t)\right.
$$

with kinetic and interaction energy components, is conserved.

The proof of Lemma 3.1 simplifies accordingly, as do the proofs of Lemma 3.2 and Lemma 3.3, now leading to the estimates

$$
\left|\int C\left(f, n_{c}\right)(p) d p\right| \leq k\left(\int f(p) d p\right)^{2}+\frac{k n_{c}}{\alpha} \int p^{2} f(p) d p
$$


respectively

$$
\int|(C(f, n)-C(g, n))(p)| d p \leq k \int\left(1+\frac{n}{\alpha} p^{2}\right)|(f-g)(p)| d p .
$$

Also the proofs of Lemma 3.4 and Lemma 3.5 simplify. From here the proof of Theorem 2.3 again becomes a simplified version of the proof of Theorem 2.1.

\section{Bibliography}

[BCEP] D. Benedetto, F. Castella, R. Esposito, M. Pulvirenti, Some considerations on the derivation of the nonlinear quantum Boltzmann equation II, J. Stat. Phys. 124, 951-966 (2006).

[E] U. Eckern, Relaxation processes in a condensate Bose gas, J. Low Temp. Phys. 54, 333-359 (1984).

[EMV] M. Escobedo, S. Mischler, M. Valle, Homogeneous Boltzmann equation in quantum relativistic kinetic theory, Electronic J. Diff. Eqns., Monograph 04 (2003).

[EPV] M. Escobedo, F. Pezzotti, M. Valle, Analytical approach to relaxation dynamics of condensed Bose gases, arXiv: 1008.0714v1.

[IT] M. Imamovic-Tomasovic, L. Kadanoff-Baym, Kinetic theory for a trapped Bose condensate gas, Thesis, Univ. Toronto, 2001.

[ITG] M. Imamovic-Tomasovic, A. Griffin, Quasi-particle kinetic equation in a trapped Bose gas at low temperature, J. Low Temp. Phys. 122, 617-655 (2001).

[HM] P. Hohenberg, P. Martin, Microscopic theory of superfluid helium, Ann. Phys. 34, 291-359 (1965).

[K] I. M. Khalatnikov, Theory of superfluidity (in Russian), Nauka, Moskva 1971.

[KD] T. R. Kirkpatrick, J. R. Dorfman, Transport in a dilute but condensed nonideal Bose gas: kinetic equations, J. Low Temp. Phys. 58, 301-331 (1985).

[KK] J. Kane, L. Kadanoff, Green's functions and superfluid hydrodynamics, Jour. math. Phys. 6, 1902-1912 (1965).

[N] A. Nouri, Bose-Einstein condensates at very low temperatures. A mathematical result in the isotropic case, Bull. Inst. Math. Acad. Sin. 2, 649-666 (2007). 
[PS] L. Pitaevski, S. Stringari, Bose-Einstein condensation, Oxford 2003.

[ST] D. Semikoz, J. Tkachev, Condensation of bosons in the kinetic regime, Phys. Rev. D, 489-502 (1997).

[S1] H. Spohn, Kinetic equations for quantum many-particle systems, Modern encyclopedia of mathematical physics, Springer 2007.

[S2] H. Spohn, Kinetics of the Bose-Einstein condensation, Physica D 239, 627-634 (2010).

[ZNG] E. Zaremba, T. Nikuni, A. Griffin, Dynamics of trapped Bose gases at finite temperatures, J. Low Temp. Phys. 116, 277-345 (1999). 